\title{
Distinct Inspiratory Rhythm and Pattern Generating Mechanisms in the preBötzinger Complex
}

\author{
Kaiwen Kam, ${ }^{1 \star}$ Jason W. Worrell, ${ }^{1 \star}$ Wiktor A. Janczewski, ${ }^{1}$ Yan Cui, ${ }^{1,2}$ and Jack L. Feldman ${ }^{1}$ \\ ${ }^{1}$ Systems Neurobiology Laboratory, Department of Neurobiology, David Geffen School of Medicine at the University of California Los Angeles, Los Angeles, \\ California 90095-1763, and 2Department of Physiology, West China School of Preclinical and Forensic Medicine, Sichuan University, Chengdu, Sichuan, \\ People's Republic of China
}

In the mammalian respiratory central pattern generator, the preBötzinger complex (preBötC) produces rhythmic bursts that drive inspiratory motor output. Cellular mechanisms initiated by each burst are hypothesized to be necessary to determine the timing of the subsequent burst, playing a critical role in rhythmogenesis. To explore mechanisms relating inspiratory burst generation to rhythmogenesis, we compared preBötC and hypoglossal (XII) nerve motor activity in medullary slices from neonatal mice in conditions where periods between successive inspiratory XII bursts were highly variable and distributed multimodally. This pattern resulted from rhythmic preBötC neural population activity that consisted of bursts, concurrent with XII bursts, intermingled with significantly smaller "burstlets". Burstlets occurred at regular intervals during significantly longer XII interburst intervals, at times when a XII burst was expected. When a preBötC burst occurred, its high amplitude inspiratory component (I-burst) was preceded by a preinspiratory component that closely resembled the rising phase of burstlets. Cadmium $(8 \mu \mathrm{m})$ eliminated preBötC and XII bursts, but rhythmic preBötC burstlets persisted. Burstlets and preinspiratory activity were observed in $\sim 90 \%$ of preBötC neurons that were active during I-bursts. When preBötC excitability was raised significantly, burstlets could leak through to motor output in medullary slices and in vivo in adult anesthetized rats. Thus, rhythmic bursting, a fundamental mode of nervous system activity and an essential element of breathing, can be deconstructed into a rhythmogenic process producing low amplitude burstlets and preinspiratory activity that determine timing, and a pattern-generating process producing suprathreshold I-bursts essential for motor output.

\section{Introduction}

In vertebrate rhythmic motor behaviors, such as breathing, chewing and locomotion, central pattern generators (CPGs) produce near synchronous bursts of action potentials in populations of neurons that determine the timing and patterning of motoneuronal activity driving participating muscles (Gossard et al., 2010). For invertebrate CPGs, mechanisms underlying generation of rhythm and pattern are closely intertwined (Selverston, 2010). In contrast, distinct rhythm and pattern generating networks are hypothesized for mammalian rhythmic motor behaviors (Feldman, 1986; McCrea and Rybak, 2008), but how, or even if, rhythm and pattern generating functions are partitioned in mammalian CPGs has not yet been determined.

In mammals, breathing is a vital, rhythmic motor behavior controlled by a CPG that operates continuously to exchange gas,

\footnotetext{
Received Aug. 28, 2012; revised April 1, 2013; accepted April 3, 2013.

Author contributions: K.K., J.W.W., W.A.J., and J.L.F. designed research; K.K., J.W.W., W.A.J., and Y.C. performed research; K.K., J.W.W., and J.L.F. analyzed data; K.K., J.W.W., and J.L.F. wrote the paper.

This work was supported by grants from the National Institutes of Health: National Heart Lung and Blood Institute and National Institute of Neurological Disorders and Stroke. We thank Grace Li for assistance with histology and immunohistochemistry and all members of the Feldman laboratory for helpful discussion.

*K.K. and J.W.W. contributed equally to this work.

The authors declare no competing financial interests.

Correspondence should be addressed to Dr. Kaiwen Kam, UCLA, Box 951763, CHS 73-152, Los Angeles, CA 90095-1763. E-mail: kwkam@mednet.ucla.edu.

DOI:10.1523/JNEUROSCI.4143-12.2013

Copyright $\odot 2013$ the authors $\quad 0270-6474 / 13 / 339235-11 \$ 15.00 / 0$
}

yet adapts rapidly and appropriately to changing physiological or environmental conditions. In the respiratory CPG in rodents, the preBötzinger complex (preBötC) is necessary for inspiratory rhythmogenesis in vitro (Smith et al., 1991) and for normal respiratory movements in vivo (Gray et al., 2001; Tan et al., 2008). Near synchronous inspiratory bursting in preBötC neurons drives activity in bulbospinal (Dobbins and Feldman, 1994) and parahypoglossal (Dobbins and Feldman, 1995; Chamberlin et al., 2007; Koizumi et al., 2008) premotoneurons projecting to spinal and XII motoneurons to produce and modulate inspiratory airflow.

preBötC bursts are critically involved in generation of patterned inspiratory motor activity and are hypothesized to be essential for rhythmogenesis. In many computational models of preBötC, inspiratory burst-initiated activation of hyperpolarizing conductances, such as $\mathrm{Ca}^{2+}$-activated $\mathrm{K}^{+}$channels or inactivation of depolarizing conductances, such as the persistent $\mathrm{Na}^{+}$ current, $\mathrm{I}_{\mathrm{NaP}}$, (Butera et al., 1999a; Rybak et al., 2004; Rubin et al., 2009), underlie rhythmogenesis. However, pharmacological blockade of these conductances does not abolish preBötC rhythmogenesis in vitro (Pena et al., 2004; Del Negro et al., 2005; Pace et al., 2007). Also inconsistent with preBötC burst-mediated rhythmogenesis is the strong modulation of inspiratory burst rhythm in vitro by extracellular $\mathrm{K}^{+}\left(\mathrm{K}_{\text {ext }}^{+}\right)$without substantial changes in inspiratory burst pattern (Del Negro et al., 2009).

Here, we test the hypothesis that preBötC rhythmogenic mechanisms are distinct from burst generating mechanisms by comparing preBötC population and XII activity in medullary 
slices from neonatal mice in conditions where the period between successive inspiratory XII bursts are highly variable (Del Negro et al., 2009) and multimodal. This temporal pattern resulted from significantly smaller amplitude preBötC bursts (burstlets) that occurred at regular intervals when a XII burst was expected, but failed to produce XII output. We characterized these events in vitro and in vivo and compared them with high amplitude preBötC bursts that produced XII bursts and multipeaked bursts that we called doublets. In preBötC single neuron and population activity, burstlets resembled preinspiratory activity, the low-level initial component of each preBötC burst. When preBötC and XII bursts were eliminated by bath-applied cadmium, rhythmic preBötC burstlets persisted. We conclude that inspiratory rhythmogenesis is primarily determined by the common mechanisms underlying burstlets and preinspiratory activity that are distinct from suprathreshold processes required to transform preBötC burstlets into preBötC bursts and bona fide XII motor output.

\section{Materials and Methods}

In vitro slice preparation and recording. The Office for the Protection of Research Subjects (University of California Animal Research Committee) approved all protocols. We used neonatal C57BL/6 mice (P0-5) of either sex for experiments in vitro. Transverse slices ( $550 \mu \mathrm{m}$ thick) were cut from the neonatal mouse brainstem, which contained the specialized rhythm-generating preBötC (Smith et al., 1991) as well as respiratory premotoneurons and XII respiratory motoneurons that discharge in phase with inspiratory rhythm (Koizumi et al., 2008). To obtain slices with the preBötC at the surface, the rostral cut was made above the first set of XII nerve rootlets at the level of the dorsomedial cell column and principal lateral loop of the inferior olive, and the caudal cut captured the obex (Ruangkittisakul et al., 2011). The medullary slice was cut in ACSF containing the following (in mM): $124 \mathrm{NaCl}, 3 \mathrm{KCl}, 1.5 \mathrm{CaCl}_{2}, 1 \mathrm{MgSO}_{4}$, $25 \mathrm{NaHCO}_{3}, 0.5 \mathrm{NaH}_{2} \mathrm{PO}_{4}$, and 30 D-glucose, equilibrated with $95 \% \mathrm{O}_{2}$ and $5 \% \mathrm{CO}_{2}, 27^{\circ} \mathrm{C}$, $\mathrm{pH} 7.4$.

Slices were perfused with $27^{\circ} \mathrm{C} \mathrm{ACSF}$ at $4 \mathrm{ml} / \mathrm{min}$ in a $0.5 \mathrm{ml}$ chamber mounted rostral side up in a fixed-stage Axioskop (Zeiss) or DMLFS (Leica) microscope set up for Koehler illumination. Bright-field or infrared-enhanced differential interference contrast (IR-DIC) video microscopy was performed using a MTI IR-series charge-coupled device (CCD) video camera (DAGE) coupled to a MTI analog camera controller (DAGE) connected to the video-in jack on a television monitor. Once placed in the recording chamber, the slice was bathed in extracellular ACSF containing 0.6-1.5 mM extracellular $\mathrm{Ca}^{2+}\left(\mathrm{Ca}_{\text {ext }}^{2+}\right)$ and 3-9 mM $\mathrm{K}_{\text {ext. }}^{+}$. In all experiments, slices were allowed to equilibrate for $30 \mathrm{~min}$ to ensure frequency and magnitude of XII and preBötC population activities reached steady-state. To study the burst/burstlet rhythm, we manipulated $\mathrm{K}_{\text {ext }}^{+}$. Changing $\mathrm{K}_{\text {ext }}^{+}$provides an experimentally advantageous means of rapidly and reversibly changing baseline membrane potential throughout the preBötC network (Del Negro et al., 2001; Johnson et al., 2001). $\mathrm{Ca}_{\text {ext }}^{2+}$ was also modulated to influence respiratory rhythm $(\mathrm{Ru}-$ angkittisakul et al., 2011). In some experiments, $3 \mathrm{~mm} \mathrm{~K} \mathrm{~K}_{\text {ext }}^{+}$and $1 \mathrm{~mm}$ $\mathrm{Ca}_{\text {ext }}^{2+}\left(3 \mathrm{mM} \mathrm{K}_{\text {ext }}^{+} / 1.0 \mathrm{mM} \mathrm{Ca}_{\text {ext }}^{2+}\right)$ were chosen as the control to maximize the relative number of burstlets, and was always tested first because rundown of the rhythm under these conditions is typically observed after 90 min (Ruangkittisakul et al., 2007). Respiratory activity reflecting suprathreshold action potential (AP) firing from populations of neurons was recorded from either XII nerve roots or as population activity from XII motoneurons and as population activity directly from the preBötC using suction electrodes and a MultiClamp 700A or 700B (Molecular Devices), filtered at $2-4 \mathrm{kHz}$ and digitized at $10 \mathrm{kHz}$. Digitized data were analyzed off-line using custom procedures written for IgorPro (Wavemetrics). Activity was full-wave rectified and digitally integrated with a Paynter filter with a time constant of $20 \mathrm{~ms}$ with either custom built electronics or using custom procedures in MATLAB (MathWorks).

Loose patch recordings were made from inspiratory preBötC neurons, selected visually with IR-DIC video microscopy in the region ventral to the semicompact division of the nucleus ambiguus, and then confirmed via recording to exhibit an inspiratory discharge pattern. These experiments were performed using either a 700A or 700B MultiClamp amplifier (Molecular Devices), and the signal was filtered at $2-4 \mathrm{kHz}$ and digitized at $10 \mathrm{kHz}$. Continuous data were acquired in current-clamp using an analog-to-digital converter (Digidata 1322 or 1440, Molecular Devices) and pCLAMP (Molecular Devices). Traces were analyzed offline using custom software written for IgorPro. Patch electrodes with a 2-3 $\mathrm{M} \Omega$ tip resistance were fabricated using a three-stage custom program on a Flaming-Brown micropipette puller (P-97, Sutter Instruments) and filled with solution containing the following (in $\mathrm{mm}$ ): 124 $\mathrm{NaCl}, 3 \mathrm{KCl}, 1.5 \mathrm{CaCl}_{2}, 1 \mathrm{MgSO}_{4}, 25 \mathrm{NaHCO}_{3}, 0.5 \mathrm{NaH}_{2} \mathrm{PO}_{4}$, and 30 D-glucose, $\mathrm{pH}$ 7.4.

In vivo recording and injections. Male Sprague Dawley rats (Charles River Laboratories), weighing 320-420 g, were anesthetized with ketamine-xylazine $(100 \mathrm{mg} / \mathrm{kg}-10 \mathrm{mg} / \mathrm{kg}$, i.p.). Atropine $(0.5 \mathrm{mg} / \mathrm{kg}$, i.p.) was given to prevent bradycardia and excessive airway secretion. Isoflurane (1-2 vol\% in air) was administered throughout the experiment. The level of anesthesia was assessed by the suppression of the withdrawal reflex and by the absence of changes in heart rate and breathing rate in response to noxious stimuli. The trachea was cannulated and respiratory flow was detected with a flow head connected to a transducer to measure airflow (GM Instruments). Coupled EMG wire electrodes (Cooner Wire) were inserted into diaphragm (DIA) muscles. Wires were connected to amplifiers (Grass Model P511; Grass Technologies) and activity was sampled at $2-4 \mathrm{kHz}$ (Powerlab 16SP, ADInstruments). The rats were placed in a supine position in a stereotaxic instrument (Kopf Instruments). The basal aspect of the occipital bone was removed to expose the ventral aspect of the medulla. To eliminate confounding effects induced by stimulation of vagal reflexes or laryngeal reflexes, rats were vagotomized by resecting a portion of the vagus nerve $(2 \mathrm{~mm})$ at midcervical level, and the larynx was denervated. Body temperature was kept constant at $37 \pm 1^{\circ} \mathrm{C}$ with a servo-controlled heating pad (Fine Science Tools). Data were recorded on a computer and analyzed using Chart 7 Pro (ADInstruments), Excel, IgorPro, and Origin7 (OriginLab) software. The absolute value of EMG signals was digitally integrated with a time constant of $0.1 \mathrm{~s}$ to calculate peak amplitude and time onset. The flow signal was high-pass filtered to eliminate DC shifts and slow drifts and digitally integrated.

Bicuculline and/or strychnine (240 $\mu \mathrm{M} ; 110 \mathrm{nl} /$ side; Sigma-Aldrich) diluted in saline were pressure injected through a sharp glass electrode ( $40 \mu \mathrm{m}$ tip diameter) into the preBötC, and, in some cases, also BötC. In burstlet experiments, bombesin ( $240 \mu \mathrm{M} ; 90 \mathrm{nl} / \mathrm{side}$; AnaSpec) was then bilaterally injected into preBötC (Janczewski et al., 2012). Coordinates were as follows (in mm): $-0.75, \pm 2.0,0.7$ for preBötC and $-0.05, \pm 2.1$, 0.6 for BötC. For stereotaxic coordinates $(x, y, z)$, the zero reference points, i.e., $(0,0,0)$, were as follows: the rostralmost rootlet of the hypoglossal nerve (RRXIIn) for the rostrocaudal axis ( $x$-axis, increase in the rostral direction), the midline for mediolateral axis ( $y$-axis), and the ventral medullary surface in dorsoventral plane (increase in a dorsal direction; Janczewski et al., 2013). A solution of fluorescent polystyrene beads ( $5 \%$ by volume of an injection; Invitrogen) was added to the injectate to mark injection sites.

Histological examination was used to determine the injection placement. At the end of each in vivo experiment, rats were transcardially perfused with $4 \%$ paraformaldehyde in phosphate buffer. The brains were collected, postfixed, and cryoprotected, and $40 \mu \mathrm{m}$ brainstem sagittal sections were cut. Specific staining for preBötC neurons (Tan et al., 2008, 2012) was used to determine whether injections were placed in the preBötC and BötC. Slides were observed under an AxioCam2 fluorescent microscope connected with AxioVision acquisition software (Zeiss).

Data analysis and statistics. Event detection, peak amplitude, duration, and area of respiratory-related activity recorded in full-wave rectified XII output or preBötC population recordings were performed using custom software written in IgorPro. Semiautomated event detection was executed using custom procedures that used multiple criteria, including slope and amplitude thresholds, to select events automatically, which were then confirmed visually by the experimenter. The onset and termination of an event (burstlet, burst, doublet) or component of an event 
(preinspiratory, I-burst) were determined after threshold detection of the event. Event onset was determined by scanning the signal backward in time from threshold crossing (marking event detection) to identify the time point when the signal intersected with baseline; the time point following the event peak at which the signal reached baseline and remained below event detection threshold for $>200 \mathrm{~ms}$ was considered event termination. Baseline was the average value of the preBötC signal over a $30 \mathrm{~s}$ window. I-burst onset in preBötC population recordings was the time at which the preBötC signal deviated from a line fit to preinspiratory activity and did not re-intersect the fitted line for $20 \mathrm{~ms}$.

Unlike intracellular recordings, suction electrode recordings lack a scale that allows comparisons across experiments, and the value of XII and preBötC burst discharge signals, i.e., measured voltage, varied significantly in absolute value between experiments. Therefore, for comparisons across experiments, the baseline was subtracted and the signal scaled to the maximum peak amplitude in the control condition for each experiment, often $9 \mathrm{~mm} \mathrm{~K}_{\text {ext }}^{+} / 1.5 \mathrm{mM} \mathrm{Ca}_{\text {ext }}^{2+}$. The amplitude and area of XII output and the amplitude and slopes of preBötC activity were then measured from the scaled signal. A peak-detection algorithm defined event amplitude as the difference between peak and baseline. Event area was computed as the integral of the scaled, full-wave rectified XII or preBötC discharge (referenced to baseline). Event duration was the time that the XII or preBötC discharge exceeded $10 \%$ of the peak amplitude. The period of inspiratory bursting in XII and preBötC was calculated as the time between the peaks of two consecutive events. The coefficient of variation $(\mathrm{CV})$ of the period was calculated by dividing the SD of the periods for a given condition by the value of the mean period for the same condition.

Event duration, amplitude and synchrony between XII and preBötC activity were criteria used to categorize detected events as burstlets, bursts, and doublets. Burstlets were events in preBötC that did not temporally overlap a XII burst. Doublets were distinguished from bursts by the presence of multiple peaks of activity. Following event detection in XII recordings, if an event displayed a second peak that exceeded $60 \%$ of the amplitude of the first peak, the event was categorized as a doublet. Additionally, two closely spaced bursts were considered a doublet based on the distribution of the period of XII output. A small peak at $<2 \mathrm{~s}$ in the distribution of periods of XII bursts was usually observed. A Gaussian was fit to this small peak and a threshold time interval was set that was its mean +3 SDs. Two bursts separated by less than this threshold were considered a doublet. This criterion was validated by looking at preBötC population activity, which usually showed that preBötC activity did not fall to baseline during the period between doublet peaks (Fig. 4A). Average traces for XII and preBötC bursts and doublets were aligned to onset of the XII burst/doublet. Average burstlet traces were aligned by the event onset.

For loose patch single neuron recording experiments when preBötC population activity was not recorded, bursts of action potentials (APs) that did not coincide with a XII burst were considered burstlets. Bursts of APs were grouped based on the distribution of interspike intervals (ISIs) where the threshold ISI separating groups was determined by a lognormal fit of the data. APs with ISIs less than threshold were grouped, whereas APs separated by ISIs longer than the threshold were considered parts of successive events. AP amplitude and spike frequency adaptation were calculated by normalizing AP amplitude and ISI to the first AP amplitude and ISI, respectively, in the burstlet, burst, or doublet. For some analyses, firing patterns during bursts were divided into a preinspiratory and an inspiratory I-burst phase, which used the sharp onset in XII burst as the boundary between the two phases.

Data are represented as mean \pm SD. Statistical significance was uniformly set at a minimum of $p<0.05$. Distributions were tested for normality using the Jarque-Bera test, which is a goodness-of-fit test, comparing the skewness and kurtosis of the test distribution with the normal distribution, where the null hypothesis is that the two distributions are not different (Jarque and Bera, 1987). Multimodality in distributions was ascertained using Hartigan's dip test for unimodality, which is a goodness-of-fit test, comparing the test distribution to a unimodal distribution that minimizes the maximum difference, where the null hypothesis is that the test distribution is unimodal (Hartigan and Hartigan, 1985). For statistical comparisons of more than two groups, an ANOVA was first performed. In most cases, a two-way repeated-measures ANOVA was used for comparisons of various parameters in different conditions and for making comparisons across different events. If the null hypothesis (equal means) was rejected, post hoc paired $t$ tests were then used for pairwise-comparisons of interest. Individual $p$ values are reported, but Holm-Bonferroni analysis for multiple-comparisons was conducted to correct for interactions between the multiple groups. For one-way and two-way ANOVAs, post hoc significance for pairwise-comparisons was analyzed using Tukey-Kramer analysis. Cumulative distribution functions (cdfs) were generated by sorting data and then binning them into $20 \times 5 \%$ bins. Distributions were compared using the KolmogorovSmirnov goodness-of-fit test.

\section{Results}

\section{Altering $\mathrm{K}_{\mathrm{ext}}^{+}$specifically changes the period of inspiratory rhythm and its variability}

In the rhythmic medullary slice preparation from perinatal rodents, the preBötC generates rhythmic inspiratory drive to XII motoneurons that leads to XII bursts (Smith et al., 1991; Ruangkittisakul et al., 2011). Changes in $\mathrm{K}_{\text {ext }}^{+}$powerfully modulate the period of XII bursts $\left(\mathrm{T}_{\mathrm{XII}}\right)$ and its associated variability (Del Negro et al., 2009; Ruangkittisakul et al., 2011), as measured by the coefficient of variation $(\mathrm{CV}=\mathrm{SD} /$ mean $)$ of $\mathrm{T}_{\mathrm{XII}}\left[\mathrm{CV}\left(\mathrm{T}_{\mathrm{XII}}\right)\right]$. We recorded XII output as $\mathrm{K}_{\text {ext }}^{+}$was lowered from 9 to $6 \mathrm{~mm}$ and then to $3 \mathrm{~mm}$, with $\mathrm{Ca}_{\text {ext }}^{2+}$ fixed at $1.5 \mathrm{~mm}$ (Fig. $1 A, B$ ). Consistent with previous findings (Del Negro et al., 2009), $\mathrm{CV}\left(\mathrm{T}_{\mathrm{XII}}\right)$ at $6 \mathrm{mM} \mathrm{K}_{\mathrm{ext}}^{+}$ was significantly greater than at either 9 or $3 \mathrm{mM} \mathrm{K}_{\text {ext }}^{+}$(Fig. $1 A$; RM ANOVA, $F_{(2,12)}=11.7, p=0.001 ; 9$ vs $6 \mathrm{mM}, p=0.0003 ; 9$ vs 3 $\mathrm{mM}, p=0.4 ; 6$ vs $3 \mathrm{~mm}, p=0.04 ; n=7)$. In contrast, mean $\mathrm{T}_{\mathrm{XII}}$ $\left(\overline{\mathrm{T}}_{\mathrm{XII}}\right)$ increased monotonically as $\mathrm{K}_{\text {ext }}^{+}$was lowered from 9 to 3 $\mathrm{mM}$ (Fig. $1 A$; RM ANOVA, $F_{(2,12)}=20.8, p=0.0001 ; 9$ vs $6 \mathrm{mM}$, $p=0.001 ; 9$ vs $3 \mathrm{mM}, p=0.001 ; 6$ vs $3 \mathrm{~mm}, p=0.003 ; n=7)$, whereas XII burst shape parameters did not change significantly from $9 \mathrm{mM} \mathrm{K}_{\text {ext }}^{+}$(Fig. 1A; RM ANOVA; amplitude: $F_{(2,12)}=3.4, p=0.07$; area: $F_{(2,12)}=1.4, p=0.3$; duration: $F_{(2,12)}=$ $9.1, p=0.004 ; 9$ vs $6 \mathrm{~mm}, p=0.2 ; 9$ vs $3 \mathrm{mM}, p=0.054 ; n=7)$. Occasionally, in both 9 and $6 \mathrm{mM} \mathrm{K}_{\text {ext }}^{+}$, longer XII bursts with two distinct peaks ("XII doublets"; see Materials and Methods) were observed (Fig. $1 B$ ) that constituted $28.2 \pm 25.1 \%$ of XII events in $9 \mathrm{mM} \mathrm{K}_{\text {ext }}^{+}$and $27.8 \pm 7.2 \%$ of XII events in $6 \mathrm{mM} \mathrm{K}_{\text {ext }}^{+}(n=7)$.

Underlying the changes in $\mathrm{T}_{\mathrm{XII}}$ and $\mathrm{CV}\left(\mathrm{T}_{\mathrm{XII}}\right)$ was a shift from a unimodal distribution of $\mathrm{T}_{\mathrm{XII}}$ in $9 \mathrm{mM} \mathrm{K}_{\mathrm{ext}}^{+}\left(\mathrm{T}_{\mathrm{XI}, 9}\right.$; Fig. $1 B, C$; see Materials and Methods; Hartigan's dip test, $p=0.7-0.9$, $n=61-95$ bursts in each of 7 slices) to a $\mathrm{T}_{\mathrm{XII}}$ distribution with multiple peaks in $6 \mathrm{mM} \mathrm{K}_{\text {ext }}^{+}$(Fig. $1 B, C$ ). These multimodal distributions were not significantly different from mixtures of Gaussians placed at integer multiples of the shortest peak (Kolmogorov-Smirnov, $p=0.08-0.7, n=98-175$ in each of 7 slices).

Multiple peaks in $6 \mathrm{mM} \mathrm{K}_{\text {ext }}^{+}$could arise from changes in $\overline{\mathrm{T}}_{\mathrm{XII}}$ over time (non-stationarity). This did not appear to be the case, as Poincaré maps of $\mathrm{T}_{\mathrm{XII}}$ in $6 \mathrm{mM} \mathrm{K}_{\mathrm{ext}}^{+}\left(\mathrm{T}_{\mathrm{XII}, 6}\right)$ had multiple clusters away from the unity line (Fig. $1 D$ ), reflecting sequential short and long $\mathrm{T}_{\mathrm{XII}, 6}$, and not a drift in the rhythm. As the modes in the distribution of $\mathrm{T}_{\mathrm{XII}, 6}$ fell at roughly integer multiples of the lowest $\mathrm{T}_{\mathrm{XII}, 6}$ peak (Figs. 1C, 2D), we hypothesized that an underlying faster rhythm originating in the preBötC was determining the longer $\mathrm{T}_{\mathrm{XII}}$. 
preBötC burstlets mediate an underlying higher frequency rhythm with lower variability

To examine whether rhythmic preBötC activity could explain the multimodal $\mathrm{T}_{\mathrm{XII}}$ in $6 \mathrm{mM} \mathrm{K}_{\text {ext }}^{+}$, we recorded simultaneously from the preBötC and the XII nerve in medullary slices (Fig. 2A,B). Inspiratorymodulated bursts of activity in slices can be recorded directly from the preBötC as neuronal population activity, which in $9 \mathrm{mM} \mathrm{K}_{\text {ext }}^{+}$is expected to be correlated (nearly) one-to-one with XII bursts (Lieske et al., 2000; Ruangkittisakul et al., 2008). We defined preBötC population activity that was concurrent with XII bursts as preBötC bursts (Lieske et al., 2000; Ruangkittisakul et al., 2008). The distribution of periods of all preBötC events in $9 \mathrm{mM} \mathrm{K}_{\text {ext }}^{+}\left(\mathrm{T}_{\text {preBötC,9 }}\right)$, normalized to $\overline{\mathrm{T}}_{\mathrm{XI}, 9}$, was similar to the distribution of $\mathrm{T}_{\mathrm{XII}, 9}$ (Fig. 2C; Kolmogorov-Smirnov, $p=$ $0.3, n=7$ slices). When $\mathrm{K}_{\text {ext }}^{+}$was lowered from 9 to $6 \mathrm{mM}, \overline{\mathrm{T}}_{\mathrm{XII}, 6}$ increased, but mean $\mathrm{T}_{\text {preBötC }}\left(\overline{\mathrm{T}}_{\text {preBötC }}\right)$ for all preBötC events did not change (paired $t$ test, $t_{(6)}=0.04$, $p=0.97, n=7$; Table 1 ). In contrast to the synchrony between preBötC and XII activity in $9 \mathrm{mM} \mathrm{K}_{\text {ext }}^{+}$, a large number of smaller preBötC population events in 6 $\mathrm{mM} \mathrm{K}_{\text {ext }}^{+}$(Table 1) were not associated with XII bursts, and instead occurred during the silent XII interburst interval (IBI; Fig. $\left.2 A,{ }^{*}\right)$. We dubbed these preBötC events burstlets to distinguish them from preBötC bursts, which were accompanied by XII bursts. In triple recordings where preBötC activity from both sides and XII activity were simultaneously measured, $99.3 \pm 0.5 \%$ of preBötC bursts and $93.1 \pm$ $4.3 \%$ of preBötC burstlets were bilaterally synchronous ( $n=6$; Fig. $2 E)$.

The distribution of $\mathrm{T}_{\text {preBötC }}$ in $6 \mathrm{~mm}$ $\mathrm{K}_{\text {ext }}^{+}\left(\mathrm{T}_{\text {preBötC,6}}\right)$ for all preBötC events, normalized to $\overline{\mathrm{T}}_{\mathrm{XII}, 9}$ for each slice, was unimodal (Hartigan's dip, $p=0.2-1, n=$ 7 slices) and significantly different from the multimodal distribution of $\mathrm{T}_{\mathrm{XII}, 6}$ (Fig. $2 C$; KolmogorovSmirnov, $p=10^{-16}, n=7$ slices), but not significantly different from the distribution of $\mathrm{T}_{\text {preBötC,9 }}$ (Fig. $2 C$; Kolmogorov-Smirnov, $p=0.8, n=7$ slices). The preBötC burstlet/burst rhythm at $6 \mathrm{~mm} \mathrm{~K}_{\text {ext }}^{+}$was significantly more regular $\left[\mathrm{CV}\left(\mathrm{T}_{\text {preBötC, } 6}\right): 0.41 \pm\right.$ $0.04]$ than that of XII bursts $\left[C V\left(T_{\mathrm{XII}, 6}\right): 0.60 \pm 0.06\right]$ and not significantly different from $\mathrm{CV}\left(\mathrm{T}_{\mathrm{XII}, 9}\right)(0.34 \pm 0.06), \mathrm{CV}\left(\mathrm{T}_{\mathrm{XII}, 3}\right)$ $(0.34 \pm 0.2)$, or $\mathrm{CV}\left(\mathrm{T}_{\text {preBötC,9}}\right)[0.38 \pm 0.07$; one-way ANOVA, $F_{(4,30)}=7.3, p=0.0003 ; \mathrm{CV}\left(\mathrm{T}_{\text {preBötC,6}, 6}\right)$ vs $\mathrm{CV}\left(\mathrm{T}_{\mathrm{XII}, 6}\right), p=0.02$; $\mathrm{CV}\left(\mathrm{T}_{\text {preBötC,6}}\right)$ vs $C V\left(\mathrm{~T}_{\mathrm{XII}, 9}\right), p=0.7 ; \mathrm{CV}\left(\mathrm{T}_{\text {preBötC,6}}\right)$ vs $\mathrm{CV}\left(\mathrm{T}_{\mathrm{XII}, 3}\right)$, $p=0.7 ; \mathrm{CV}\left(\mathrm{T}_{\text {preBötC,6}, 6}\right)$ vs $\left.\mathrm{CV}\left(\mathrm{T}_{\text {preBötC,9}}\right), p=1 ; n=7\right]$. Further, $\mathrm{T}_{\mathrm{XII}, 6}$ increased monotonically with the number of burstlets during the IBI. $\overline{\mathrm{T}}_{\mathrm{XII}, 6}$ when $0,1,2$, or 3 burstlets were observed in preBötC were not significantly different from the corresponding integer multiple, e.g., 1, 2, 3, or 4, respectively, of $\overline{\mathrm{T}}_{\text {preBötC,6}}$ (Fig. $2 D$; Student's $t$ test; 1 burstlet: $t_{(6)}=0.007, p=1 ; 2$ burstlets: $t_{(6)}$
B

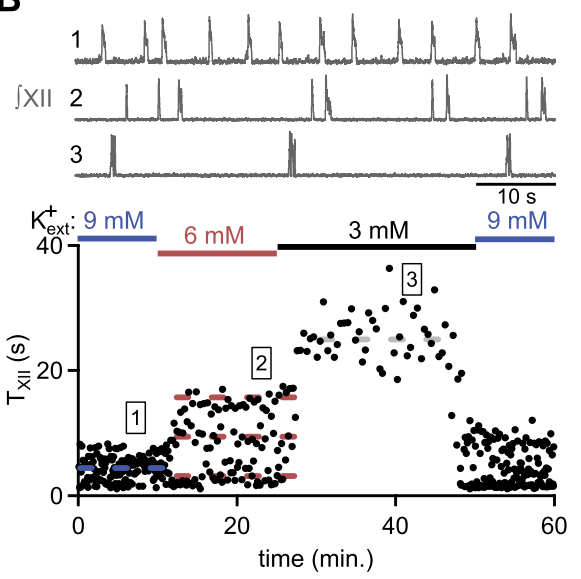

D

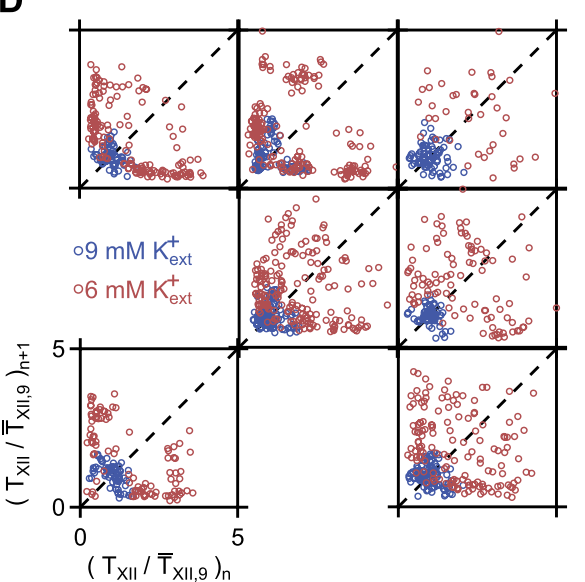

Figure 1. Increase in rhythmic variability arises from a multimodal distribution of periods. $A$, Normalized integrated XII burst amplitude (amp.), area, and duration (dur.) did not change significantly as $\mathrm{K}_{\text {ext }}^{+}$was decreased from 9 to $6 \mathrm{~mm}$ to $3 \mathrm{~mm}$, then back to

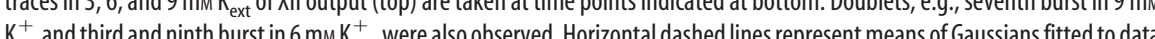
in each condition. In $6 \mathrm{~mm} \mathrm{~K}_{\text {ext }}^{+}$intervals are present at 9 and $15 \mathrm{~s}$, approximately three and five times the lowest $\mathrm{T}_{\mathrm{XII}}$ of $3 \mathrm{~s}$. C, Increase

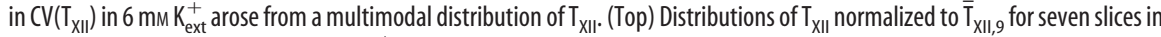
$9 \mathrm{~mm}$ (left, blue) and $6 \mathrm{~mm}$ (right, red) $\mathrm{K}_{\text {ext }}^{+}$Vertical dashed lines represent integer multiples of average value of shortest peak. (Bottom) Averaged cdfs. Data are mean \pm SD (note, SDs are too small to see in most cases). D, Multimodal distribution did not result from non-stationarity ( $n=7$ slices). Poincaré plots of $\mathrm{T}_{\mathrm{XII}} / \overline{\mathrm{T}}_{\mathrm{XII}, 9}$ show single clusters near the unity line (dashed line) in $9 \mathrm{~mm}$ $\mathrm{K}_{\text {ext }}^{+}$(blue), indicating regularity in the rhythm throughout each experiment. In $6 \mathrm{~mm} \mathrm{~K}_{\text {ext }}^{+}$(red), a quasiperiodic distribution is evident with clusters located away from unity line. Clusters near axes indicate alternating short and long $\mathrm{T}_{\mathrm{XII}}$.

$=2.1, p=0.08 ; 3$ burstlets: $\left.t_{(6)}=1.5, p=0.2 ; n=7\right)$. Burstlet/ burst patterns persisted when $\mathrm{K}_{\text {ext }}^{+}$was lowered to $3 \mathrm{~mm}$ (Fig. $2 A$ ), as well as under more physiological cation concentrations when $\mathrm{K}_{\text {ext }}^{+}$and $\mathrm{Ca}_{\text {ext }}^{2+}$ were lowered to 3 and $1 \mathrm{~mm}$, respectively (Fig. 3E; Table 1; Ruangkittisakul et al., 2011). Thus, preBötC burstlets and bursts together constituted a regular rhythm underlying the multimodal distribution of $\mathrm{T}_{\mathrm{XII}, 6}$.

Preinspiratory activity links burstlets and bursts, constituting an obligatory burstlet-like rhythmogenic process that triggers pattern generating activity

To examine whether a burstlet-like component could be observed in preBötC bursts, we compared the shape of preBötC bursts and burstlets in $6 \mathrm{mM} \mathrm{K}_{\text {ext }}^{+}$. The peak amplitudes of preBötC bursts were significantly larger than that of burstlets (Figs. $2 A, 3 A-C$; paired $t$ test, $\left.t_{(6)}=7.5, p=0.0003, n=7\right)$, whereas 
A

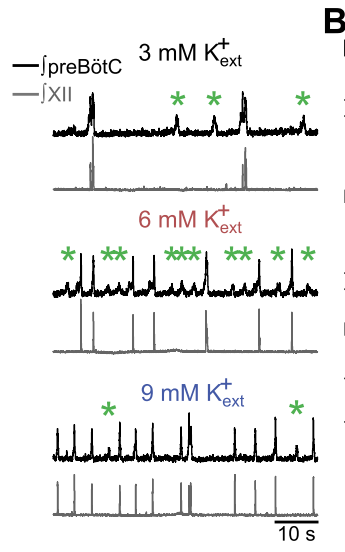

C
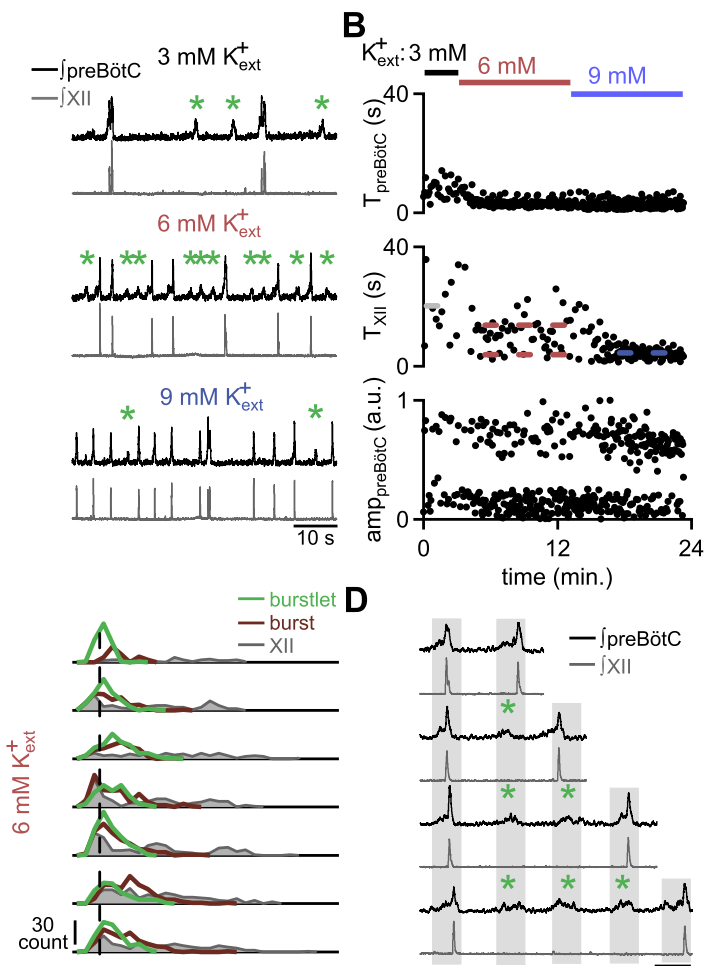

D
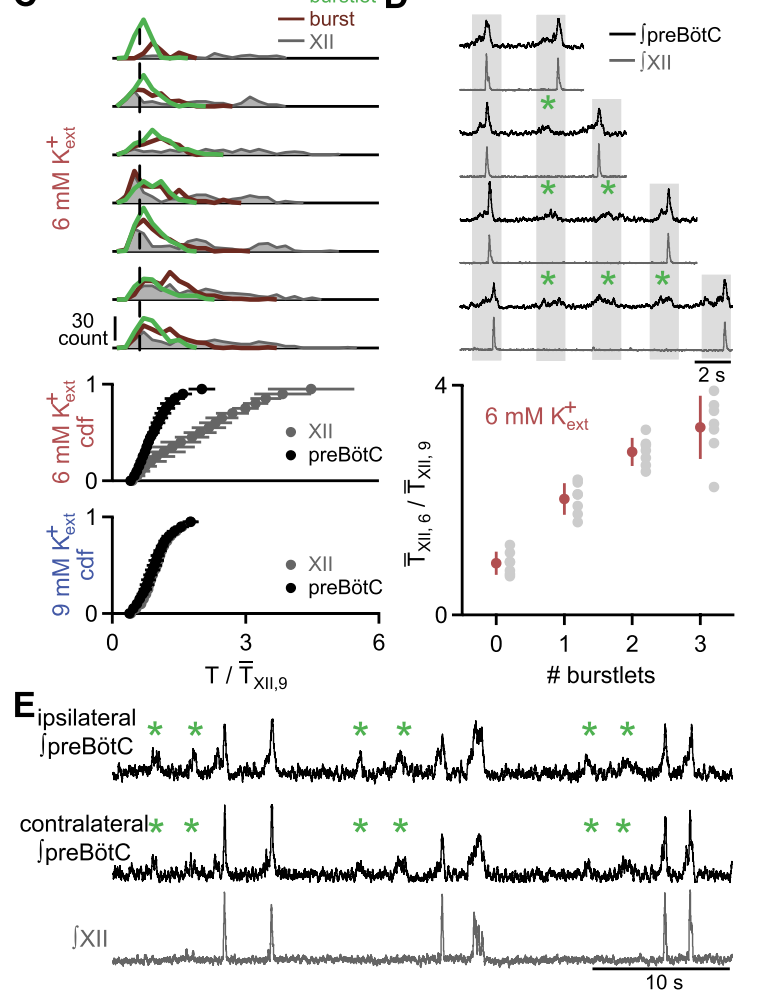

Figure 2. Higher frequency burstlets in preBötC underlie the multimodal distribution of $\mathrm{T}_{\mathrm{XII}}$. $A$, Representative traces of simultaneous XII output (gray) and preBötC (black) recordings in 9 , 6 , and $3 \mathrm{~mm} \mathrm{~K} \mathrm{ext}_{\text {et }}^{+}$hhow bursts (large amplitude preBötC events that generate XII output) and burstlets (smaller amplitude preBötC events that do not result in XII activity; indicated with green asterisk). XII and preBötC doublets, e.g., fifth burst in $6 \mathrm{~mm} \mathrm{~K}_{\mathrm{ext}}^{+}$and eighth burst in $9 \mathrm{~mm}$ $\mathrm{K}_{\mathrm{ext}}^{+}$were also observed. $\boldsymbol{B}$, Representative time course of XII output and preBötC population recording in 9, 6, and $3 \mathrm{~mm} \mathrm{~K}_{\text {ext }}^{+}$showing $\mathrm{T}_{\text {preBötc }}\left(\right.$ top), $\mathrm{T}_{\mathrm{XII}}$ (middle), and amplitudes of preBötC events, i.e., burstlets and bursts (amp preBötc; bottom) in a.u. $\mathrm{T}_{\text {preBötc }}$ shows lower variability compared with $\mathrm{T}_{\mathrm{XIl}} \cdot \mathrm{Amp}_{\text {preBötc }}$ is bimodal. C, Top, Distributions of burstlet $\rightarrow$ next event (burstlet, burst, or doublet; green) and burst $\rightarrow$ next event (maroon) $\mathrm{T}_{\text {preBötc, }, 6}$ and $\mathrm{T}_{\mathrm{XII,}, 6}$ (gray), all normalized to $\overline{\mathrm{T}}_{\mathrm{XII}, 9}$ for individual slices. Dotted line represents average value of shortest peak in $\mathrm{T}_{\mathrm{XII}}$ distribution. C, Bottom, Cdfs of $\mathrm{T}_{\mathrm{XII}}$ (gray) and $\mathrm{T}_{\text {preBötc }}$ (black) in 9 and $6 \mathrm{~mm} \mathrm{~K}_{\text {ext }}^{+}$show significant overlap in $9 \mathrm{~mm} \mathrm{~K}_{\text {ext, }}^{+}$whereas the distribution of $\mathrm{T}_{\text {preBötc }}$ is significantly different from that of $\mathrm{T}_{\mathrm{XII}}$ in $6 \mathrm{~mm} \mathrm{~K}_{\mathrm{ext}}^{+}$. Data are mean $\pm \mathrm{SD}(n=7)$. D, Top, Representative traces of preBötC (black) and XII (gray) recordings with different numbers of burstlets during the IBI show that $\mathrm{T}_{\mathrm{XII}}$ increased monotonically with the number of burstlets. Gray bars indicate when the next burstlet was expected (green asterisk indicate burstlets). D, Bottom, $\overline{\mathrm{T}}_{\mathrm{XII}, 6} / \overline{\mathrm{T}}_{\mathrm{XI}, 9}$ as a function of the number of burstlets in the IBI demonstrate that $\overline{\mathrm{T}}_{\mathrm{XII}, 6}$ increases monotonically with the number of burstlets ( $n=7$; bottom). Means for each slice are depicted as gray circles. $\boldsymbol{E}$, Simultaneous recording of XII (gray) and ipsi- and contralateral preBötC (black) population activity show that burstlets (green asterisk) are bilaterally synchronous. Doublets, e.g., fourth burst, were also observed bilaterally in preBötC.
Table 1. Effects of changing extracellular ions on preBötC rhythm and pattern

\begin{tabular}{lll}
\hline Condition & $\mathrm{T}_{\text {preBötc }}(s)$ & Burstlets (\%) \\
\hline $9 \mathrm{~mm} \mathrm{~K}_{\text {ext }}^{+} / 1.5 \mathrm{~mm} \mathrm{Ca}_{\text {ext }}^{2+}(n=7)$ & $3.46 \pm 0.81$ & $18.4 \pm 10.2$ \\
$6 \mathrm{mM} \mathrm{K}_{\text {ext }}^{+} / 1.5 \mathrm{~mm} \mathrm{Ca}_{\text {ext }}^{2+}(n=7)$ & $3.46 \pm 0.46$ & $51.6 \pm 9.9$ \\
$3 \mathrm{mM} \mathrm{K}_{\text {ext }}^{+} / 1.5 \mathrm{~mm} \mathrm{Ca}_{\text {ext }}^{2+}(n=7)$ & $5.96 \pm 1.39$ & $70.0 \pm 8.7$ \\
$3{\mathrm{~mm} \mathrm{~K} \mathrm{ext}^{+}}^{+} / 1.0 \mathrm{~mm} \mathrm{Ca}_{\text {ext }}^{2+}(n=7)$ & $4.13 \pm 1.11$ & $64.0 \pm 6.3$ \\
$8 \mu \mathrm{M} \mathrm{Cd}_{\text {ext }}^{2+} / 3 \mathrm{~mm} \mathrm{~K}_{\text {ext }}^{+} / 1.0 \mathrm{~mm} \mathrm{Ca}_{\text {ext }}^{2+}(n=4)$ & $2.38 \pm 1.30$ & $100 \pm 0$ \\
\hline
\end{tabular}

$\mathrm{T}_{\text {preBötc }}$ and the percentage of preBötC events that are burstlets in conditions where either $\mathrm{K}_{\text {ext }}^{+} \mathrm{C}_{\text {ext }}^{2+}$, or $\mathrm{Cd}_{\mathrm{ext}}^{2+}$ is changed. The number of slices $(n)$ is displayed in parentheses. Data are mean $\pm S D$.

preBötC burst duration $(0.72 \pm 0.17 \mathrm{~s})$ did not differ significantly from preBötC burstlet duration $\left(1.15 \pm 0.34 \mathrm{~s}\right.$; paired $t$ test, $t_{(6)}=$ $2.3, p=0.06 ; n=7)$. In $5 / 7$ slices, the distribution of preBötC burstlet and burst peak amplitudes was bimodal (Hartigan's dip, $p=2 \times 10^{-5}-0.002, n=5$ ), whereas the distributions for the other two slices showed a second peak by visual inspection that was not sufficiently distinct to reach statistical significance for multimodality (Fig. 3B). This suggests that mechanisms underlying the significantly greater amplitude of preBötC bursts were distinct from those generating burstlets.

Unlike inspiratory XII bursts, preBötC bursts in 9 and $6 \mathrm{~mm}$ $\mathrm{K}_{\text {ext }}^{+}$did not arise abruptly or smoothly from baseline. Rather, we identified two components in preBötC bursts. A high amplitude inspiratory burst component ("preBötC I-burst") initially led XII bursts ( $\left.9 \mathrm{mM} \mathrm{K}_{\text {ext }}^{+}: 28 \pm 14 \mathrm{~ms} ; 6 \mathrm{mM} \mathrm{K}_{\text {ext }}^{+}: 60 \pm 17 \mathrm{~ms} ; n=7\right)$, but peaked almost concurrently with XII bursts (preBötC $\rightarrow$ XII peak lag, positive indicates preBötC peak precedes XII peak; $9 \mathrm{~mm} \mathrm{~K}_{\text {ext: }}^{+}$: $\left.8 \pm 12 \mathrm{~ms} ; 6 \mathrm{~mm} \mathrm{~K}_{\mathrm{ext}}^{+}: 3 \pm 10 \mathrm{~ms} ; n=7\right)$. The preBötC I-burst was preceded by low-level "preinspiratory" activity lasting $337 \pm 121$ $\mathrm{ms}$ in $6 \mathrm{mM} \mathrm{K}_{\mathrm{ext}}^{+}$, which was significantly longer than preinspiratory activity in $9 \mathrm{~mm} \mathrm{~K} \mathrm{~K}_{\text {ext }}^{+}\left(176 \pm 66 \mathrm{~ms}\right.$; paired $t$ test, $t_{(6)}=3.2$, $p=0.02 ; n=7$; Fig. $3 A, D)$.

Preinspiratory activity resembled the rising phase of burstlets. In $6 \mathrm{mM} \mathrm{K}_{\mathrm{ext}}^{+}$, the rising slope of preinspiratory activity, corresponding to the rate of increase in preBötC population activity, was not different from the rising slope of burstlets, but was significantly different from the slope of preBötC I-bursts (Fig. 3D; RM ANOVA, $F_{(2,12)}=63.6, p=4 \times 10^{-7}$; burstlet vs preinspiratory, $p=0.1$; burstlet vs I-burst, $p=0.0004$; preinspiratory vs I-burst, $\left.p=5 \times 10^{-5} ; n=7\right)$. The peak burstlet amplitude $[0.19 \pm 0.10$ arbitrary units (a.u.) $]$ was also not significantly different from peak preinspiratory amplitude (Fig. 3D; $0.19 \pm$ 0.13 a.u.; paired $t$ test, $t_{(6)}=0.04, p=1 ; n=7$ ). The congruence between burstlets and preinspiratory activity in initial slope and peak amplitude, along with the unimodal distribution of $\mathrm{T}_{\text {preBötC}}$, suggests a common rhythmogenic process underlying both events. Furthermore, these data imply that preBötC bursts were not unitary events, but arose from a rhythmogenic burstlet/preinspiratory component that always preceded a mechanistically distinct preBötC I-burst component.

To determine whether preBötC I-burst generation constituted a process distinct from rhythmogenic burstlet activity, we attempted to selectively block I-bursts. Bath-applied cadmium $\left(\mathrm{Cd}_{\text {ext }}^{2+} ; 8 \mu \mathrm{M}\right)$ abolished preBötC and XII bursts in $3 \mathrm{~mm} \mathrm{~K} \mathrm{~K}_{\text {ext }}^{+}$and $1 \mathrm{mM} \mathrm{Ca}_{\text {ext }}^{2+}$ (Fig. $3 E$, F; paired $t$ test; preBötC/XII burst frequency: $t_{(4)}=3.1, p=0.04$; preBötC burst amplitude: $t_{(4)}=41.1, p=2 \times$ $10^{-6} ; n=5$ ), while preBöt $C$ burstlets continued, with no change in frequency or burstlet amplitude (Fig. $3 E$, F; Table 1; paired $t$ test; frequency: $t_{(4)}=0.9, p=0.4$; amplitude: $t_{(4)}=0.5, p=0.6$; $n=5) . \mathrm{CV}\left(\mathrm{T}_{\text {preBötC }}\right)$ in control conditions $(0.41 \pm 0.11)$ and in $\mathrm{Cd}_{\text {ext }}^{2+}(0.25 \pm 0.13)$ also were not significantly different (paired $t$ test, $t_{(4)}=2.3, p=0.08, n=5$ ). preBötC burstlets appeared to be the 
result of a state where preBötC I-burst generation failed, either sporadically, when $\mathrm{K}_{\text {ext }}^{+}$ was lowered, or completely, when $\mathrm{Cd}_{\text {ext }}^{2+}$ was added, whereas an underlying rhythmogenic mechanism continued relatively unperturbed.

An apparent threshold for transmission of activity to XII output filters preBötC population activity

Low-level preBötC activity, i.e., burstlets, did not generate XII output, suggesting that preBötC population activity below a certain threshold was unable to propagate to premotoneurons and motoneurons to trigger XII activity. Consistent with a transmission threshold, preBötC activity concurrent with XII doublets ("preBötC doublets"; Fig. 2A,E) usually displayed longer plateaus or multiple peaks that maintained activity during the XII interpeak pause (Fig. $4 A$ ), although with sufficiently long pauses, preBötC activity could also return to baseline. Doublet $\rightarrow$ next event (burstlet, burst, or doublet) $\mathrm{T}_{\text {preBötc }}$ were significantly longer than burst $\rightarrow$ next event and burstlet $\rightarrow$ next event $T_{\text {preBötC }}$ in $6 \mathrm{~mm}$ $\mathrm{K}_{\text {ext }}^{+}$(Fig. $4 B$; RM ANOVA, $F_{(2,12)}=18.5$, $p=0.0002$; burstlet vs doublet, $p=0.003$; burst vs doublet, $p=0.006 ; n=7$ ) and longer than burst $\rightarrow$ next event $\mathrm{T}_{\text {preBötC }}$ in $9 \mathrm{mM}$ $\mathrm{K}_{\text {ext }}^{+}$(Fig. $4 B$; paired $t$ test, $t_{(6)}=3.3, p=$ $0.02, n=7)$. Thus, following a doublet there was a longer delay to the next preBötC burstlet, burst, or doublet.

We hypothesized that increasing excitability in premotoneurons and/or motoneurons could lower this transmission threshold to allow preBötC activity to appear in motor output. As increases in $\mathrm{K}_{\mathrm{ext}}^{+}$ reduced the occurrence of burstlets, we tested whether we could generate burstlet activity in XII output by lowering $\mathrm{Ca}_{\mathrm{ext}}^{2+}$ to increase excitability (Ruangkittisakul et al., 2011). When $\mathrm{K}_{\text {ext }}^{+}$and $\mathrm{Ca}_{\text {ext }}^{2+}$ were lowered to 3 and $1 \mathrm{mM}$, respectively (Fig. $3 E$; Ruangkittisakul et al., 2011), rhythmic activity recorded in XII output and preBötC consistently showed a preBötC burstlet/ burst pattern similar to that seen in $6 \mathrm{~mm}$ $\mathrm{K}_{\text {ext }}^{+} / 1.5 \mathrm{mM} \mathrm{Ca}_{\text {ext }}^{2+}$ (Table 1 ). Increasing excitability by further lowering $\mathrm{Ca}_{\text {ext }}^{2+}$ to $0.6-0.8 \mathrm{~mm}$ produced smaller events in XII output ("XII burstlets") that were synchronous with preBötC burstlets (Fig. 4C). The amplitude of XII burstlets was significantly lower than XII burst amplitude (Fig. 4D; paired $t$ test, $\left.t_{(3)}=3.7, p=0.03, n=4\right)$. However, the amplitude of preBötC burstlets associated with XII burstlets (burstlet + XII) did not differ significantly from the amplitude of preBötC burstlets not associated with XII burstlets (burstlet-XII) in the same condition or preBötC burstlet amplitude in the control $3 \mathrm{~mm} \mathrm{~K}_{\text {ext }}^{+} / 1 \mathrm{mM} \mathrm{Ca}_{\text {ext }}^{2+}$ condition; these preBötC burstlet amplitudes all remained significantly lower than preBötC burst amplitude (Fig. 4D; RM ANOVA, $F_{(3,9)}=52.8, p=5 \times 10^{-6}$; control burstlet vs burstletsymbols.
B
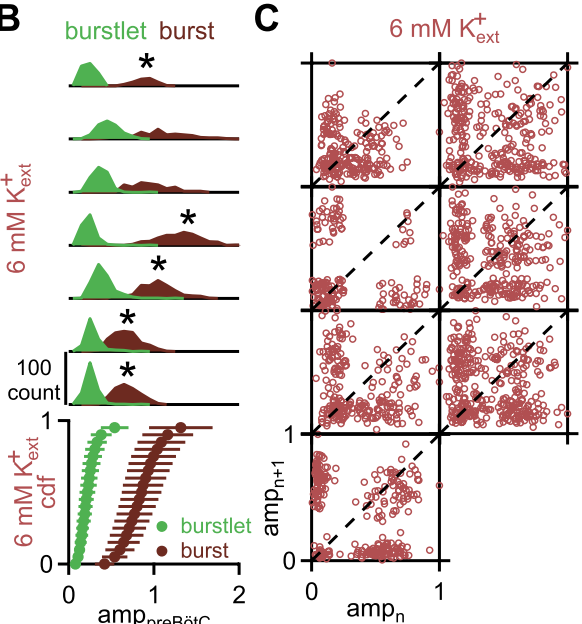

E control - jpreBötC $\mathbf{F}$

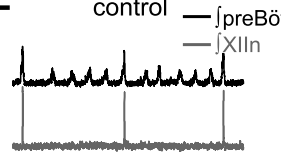

$8 \mu \mathrm{M} \mathrm{Cd} \mathrm{ext}^{2+}$
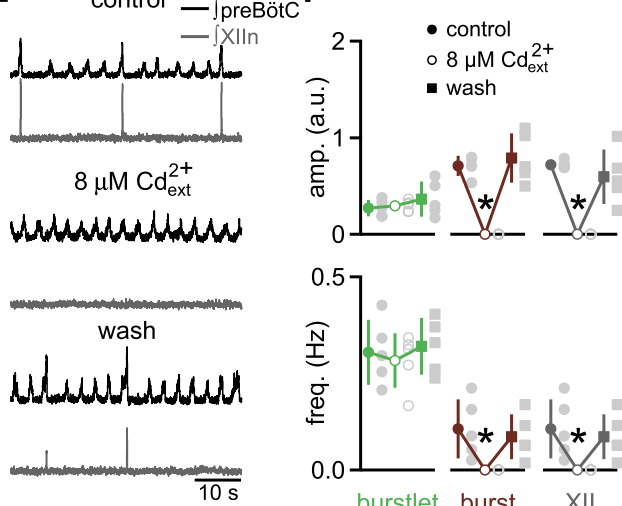

Figure 3. Mechanisms underlying preBötC burst and burstlet generation are distinct and separable. $\boldsymbol{A}$, Representative traces of preBötC (black) and XII (gray) recordings during burstlets (top) or bursts (bottom) in $6 \mathrm{~mm} \mathrm{~K}+$ ext highlight preinspiratory activity (arrows) preceding the preBötC I-burst. Red line indicates onset of preBötC burstlet and burst. $\boldsymbol{B}, \ln 6 \mathrm{~mm} \mathrm{~K} \mathrm{~K}_{\text {ext' }}^{+}$burstlet amplitude is significantly smaller than burst amplitude $(n=7)$. $\boldsymbol{B}$, Top, Distributions of preBötC burstlet (green) and burst (maroon) amplitudes show a clear bimodal distribution that justifies this categorization. Asterisk indicates distribution is significantly , $p<0.05$. B, Bottom, Cdfs of amplitude of preBötC events (amp preBöt $)$ show significantly different burstlet and burs $a_{\text {preBötc. }}$ Data are mean $\pm S D$. C, Representative Poincaré plots of amp preBötc in $6 \mathrm{~mm} \mathrm{~K}_{\text {ext. }}^{+}$. Small and large amplitude preBötC events, likely representing burstlets and bursts, respectively, are temporally intermingled, and amplitudes are not continuously in burstlets (green) and preinspiratory rise (maroon) of bursts. Vertical dotted line indicates start of XII inspiratory burst and traces indicates division of preBötC bursts into preinspiratory and I-burst components. Half-arrow indicates onset of preBötC burstlet and burst. D, Bottom, Average slopes for rising phase of burstlets and preinspiratory and I-burst components of preBötC bursts in a.u. Preinspiratory rate of rise does not differ significantly from that of burstlets, but is significantly less than -burstrate of rise. Data are mean $\pm S D\left({ }^{*} p<0.05 ; n=7\right)$ with means for each slice as gray circles. $\boldsymbol{E}$, Cadmium $\left(\mathrm{Cd}_{\text {ext }}^{2+}\right)$ abolishes preBötC bursts and all XII output while burstlets continue. Representative traces showing effects of $8 \mu \mathrm{m} \mathrm{Cd}_{\text {ext }}^{2+}$ on preBötC (black) and XII (gray) recording. F, Average data show amplitude and frequency of preBötC burstlets (green) and preBötC (maroon) and XI

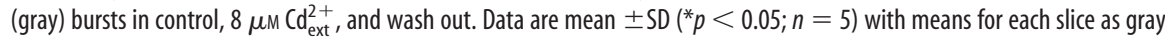

XII, $p=0.6$; control burstlet vs burstlet $+\mathrm{XII}, p=0.9$; control burstlet vs burst, $p=0.0008$; burstlet-XII vs burstlet $+\mathrm{XII}, p=$ 0.3 ; burstlet-XII vs burst, $p=0.005$; burstlet + XII vs burst, $p=$ $0.0009 ; n=4)$.

\section{Most preBötC inspiratory-modulated neurons fire during both burstlets and bursts}

To determine how the activity of single neurons could sum to produce preBötC population activity, inspiratory-modulated preBötC neurons were recorded in loose patch configuration in 3 $\mathrm{mM} \mathrm{K}_{\text {ext }}^{+} / 1 \mathrm{mM} \mathrm{Ca}_{\text {ext }}^{2+}(n=18)$ while monitoring XII output and/or contralateral preBötC population activity. Most, but not all, of these neurons generated APs during both bursts and burst- 
A

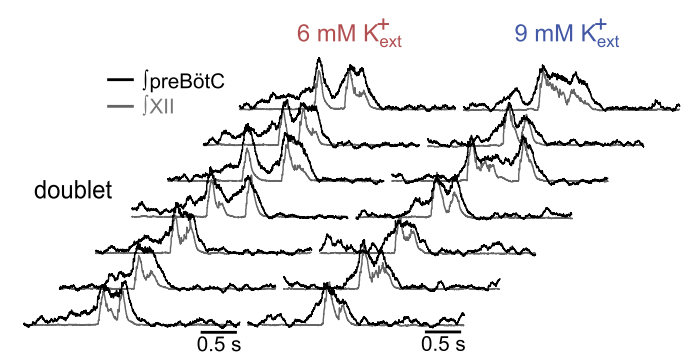

C

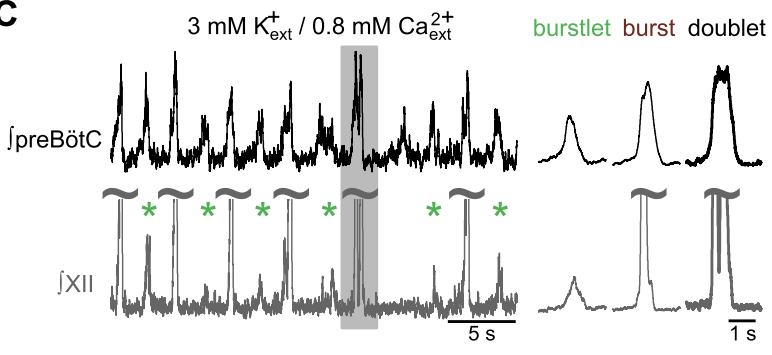

B

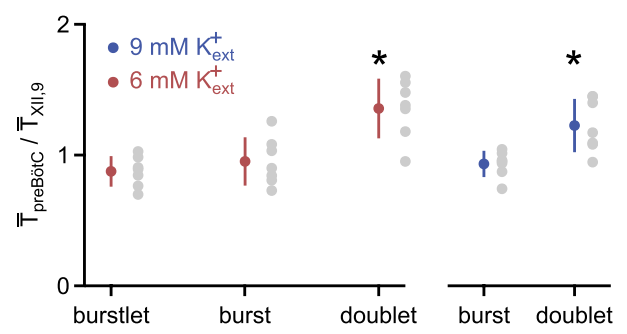

D

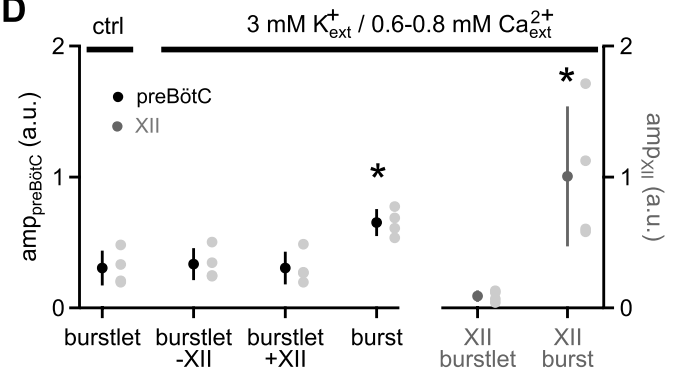

Figure 4. Patterning of XII output is not restricted to preBötC burst generation. $A$, Representative traces of simultaneous recordings of preBötC (black) and XII (gray) activity during doublets in 9 and $6 \mathrm{~mm} \mathrm{~K}_{\text {ext }}^{+}$demonstrating that preBötC activity during doublets does not drop to baseline during the pause between peaks in XII output. $\boldsymbol{B}$, Burstlet $\rightarrow$ next event, burst $\rightarrow$ next event, and doublet $\rightarrow$ next event $\overline{\mathrm{T}}_{\text {preBötc }}$, normalized to $\overline{\mathrm{T}}_{\mathrm{XII}, 9}$. In 6 and $9 \mathrm{~mm} \mathrm{~K} \mathrm{~K}_{\text {ext' }}^{+}$doublet $\rightarrow$ next event $\overline{\mathrm{T}}_{\text {preBötc }}$ is longer than both burstlet $\rightarrow$ next event and burst $\rightarrow$ next event $\overline{\mathrm{T}}_{\text {preBöt },}$, whereas burstlet $\rightarrow$ next event and burst $\rightarrow$ next event $\overline{\mathrm{T}}_{\text {preBötc }}$ do not differ. Data are mean \pm SD ( ${ }^{*} p<0.05 ; n=7$ ) with means for each slice as gray circles. $C$, Left, Respiratory-related rhythm in 3 mm $\mathrm{K}_{\text {ext }}^{+}$ and $0.8 \mathrm{~mm} \mathrm{Ca} \mathrm{Cext}_{\mathrm{e}}^{2+}$ had burstlets (indicated by green asterisk) and doublets (indicated by gray box), in both preBötC (black) and XII output (gray). C, Right, Average XII (gray) and preBötC (black) traces during burstlets, bursts, and doublets show the differences in amplitude. XII bursts and doublets are trimmed (represented by $\sim$ ), in both left and right, to show XII burstlets. $D$, Amplitudes of

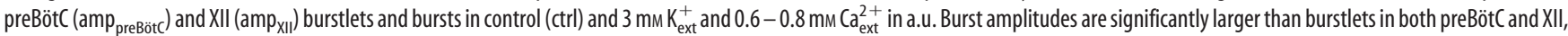
whereas amplitudes of preBötC burstlets associated with XII burstlets (burstlet + XII) do not differ significantly from control preBötC burstlet amplitude or amplitudes of burstlets not associated with XII burstlets (burstlet-XII) in $3 \mathrm{~mm} \mathrm{~K}_{\text {ext }}^{+}$and $0.6-0.8 \mathrm{~mm} \mathrm{Ca}_{\text {ext }}^{2+}$. Data are mean $\pm \mathrm{SD}\left({ }^{*} p<0.05 ; n=4\right)$ with means for each slice as gray circles.

lets $(n=16 / 18,89 \%$; Fig. $5 A)$. In 2/18 (11\%) neurons, APs were generated only during bursts (Fig. $5 B$ ). No neurons (0/18) fired only during burstlets. To test for pacemaker properties, neurons that fired during both bursts and burstlets were synaptically isolated with blockers of fast excitatory and inhibitory transmission (10 $\mu \mathrm{M}$ CPP, $10 \mu \mathrm{M}$ NBQX, $1 \mu \mathrm{M}$ strychnine, $100 \mu \mathrm{M}$ picrotoxin). With fast synaptic transmission blocked, a small percentage of these neurons continued to burst (2/16, 13\%; Fig. $5 C)$. This percentage is similar to that of all inspiratory-modulated neurons identified as having pacemaker properties (Thoby-Brisson and Ramirez, 2001; Del Negro et al., 2005).

For neurons that fired during both bursts and burstlets, the duration of AP firing during burstlets was significantly shorter than during bursts (Figs. 5, 6; burstlets: $462 \pm 43 \mathrm{~ms}$; bursts: $665 \pm 64 \mathrm{~ms}$; paired $t$ test, $\left.t_{(15)}=5, p=0.0002 ; n=16\right)$. There was a trend toward a reduction in AP amplitude during burstlets to $63 \pm 4 \%$ of the amplitude of the first AP (Figs. 5, 6). During bursts, however, AP amplitude was significantly attenuated to $39 \pm 4 \%$ of the amplitude of the first AP (Figs. 5, 6; paired $t$ test, $\left.t_{(15)}=7, p=4 \times 10^{-6}, n=16\right)$. In some cases, APs decreased in amplitude or even transiently disappeared during the middle of a burst before returning (Figs. 5, 6). These events often corresponded to doublets, as seen in both (contralateral) preBötC population recording and XII output (Figs. 5, 6). For each neuron, the number of APs varied widely from burstlet to burstlet (Fig. $6 A$; range: $2-37$ spikes; $C V: 0.58 \pm 0.05 ; n=16$ ) and less so from burst to burst (Fig. $6 A$; range: $2-43$ spikes; CV: $0.37 \pm 0.02$; $n=16$ ).

Because population data suggested a common process underlying burstlets and preinspiratory activity, we divided spiking behavior during preBötC bursts into preinspiratory and preBötC I-burst components and compared firing during these phases with burstlet spiking. The average number and maximum fre- quency of APs during burstlets were not significantly different from these values during the preinspiratory phase, but both were significantly lower than these values during the preBötC I-burst (Fig. $6 B$; one-way ANOVA; AP number: $F_{(2,44)}=11.9, p=8 \times$ $10^{-5}$; burstlet vs preinspiratory, $p=0.6$; burstlet vs I-burst, $p=$ 0.003 ; preinspiratory vs I-burst, $p=9 \times 10^{-5}$; maximum AP frequency: $F_{(2,44)}=20.1, p=6 \times 10^{-7}$; burstlet vs preinspiratory, $p=1$; burstlet vs I-burst, $p=7 \times 10^{-6}$; preinspiratory vs I-burst, $\left.p=6 \times 10^{-6} ; n=16\right)$. The similarities in firing pattern between burstlets and preinspiratory activity and their significant differences from preBötC I-bursts are consistent with the population data.

\section{Behavioral correlates of XII burstlets, bursts, and doublets are observed in vivo under specific conditions}

To examine whether burstlets, bursts, and doublets were representative of breathing in vivo, the respiratory pattern [airflow and diaphragm EMG activity $\left(\mathrm{DIA}_{\mathrm{EMG}}\right)$ ] were recorded in anesthetized adult rats. Most airway and pulmonary sensory feedback was eliminated by laryngeal denervation, tracheostomy below the larynx, and then later during an experiment, vagotomy. In anesthetized, vagotomized adult rats, the shape and timing of bursts in DIA $_{\mathrm{EMG}}$ were uniform, and we did not see small burstlet-like events or any double-peaked events in $\mathrm{DIA}_{\mathrm{EMG}}$ (Fig. 7A, left). In vitro, increasing excitability by lowering $\mathrm{Ca}_{\text {ext }}^{2+}$ allowed burstlet activity to leak through to XII output, producing smaller events that were synchronous with preBötC burstlets (Fig. 4C). In an effort to evoke burstlets in vivo comparable to those seen in vitro when we increased excitability, we disinhibited the preBötC and BötC by local injection of bicuculline (Janczewski et al., 2013). Excitability was further increased with bilateral injection into the preBötC of bombesin (Janczewski et al., 2012), a peptide that depolarizes preBötC neurons (Gray et al., 1999; Iniushkin and 
A



B
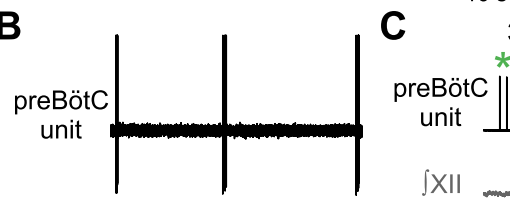

$3 \mathrm{mM} \mathrm{K}_{\text {ext }}^{+} / 1.0 \mathrm{mM} \mathrm{Ca}_{\text {ext }}^{2+}$

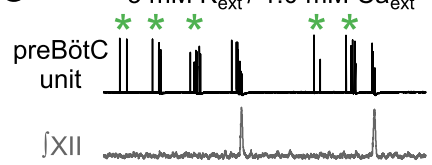

NBQX / CPP /
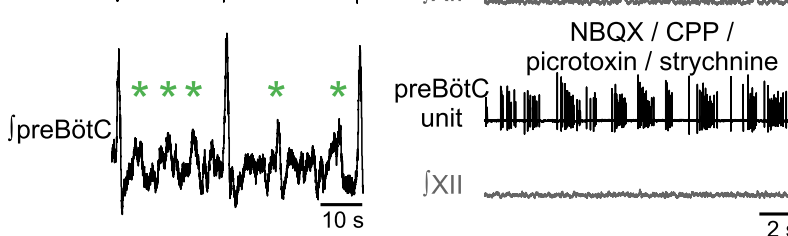

unit

JXII
Figure 5. Single unit recordings show individual preBötC neurons fire during burstlets and bursts. $\boldsymbol{A}$, Left, Representative traces of XII (gray), preBötC population activity (black) and a preBötC neuron (black) in $3 \mathrm{~mm} \mathrm{~K}_{\text {ext }}^{+} / 1 \mathrm{~mm} \mathrm{Ca}_{\text {ext }}^{2+}$, indicating spiking during both burstlets (green asterisk), bursts, and doublets (gray box). $A$, Right, Expanded time scales for recordings during a burstlet, burst, and doublet for the experiment shown demonstrate changes in duration, AP number, frequency, and amplitude between preBötC event categories. $\boldsymbol{B}$, Representative traces of preBötC neuron and contralateral preBötC population activity showing a burst only neuron (green asterisk indicate burstlets). C, Neuron showing burstlet (indicated by green asterisk) and burst activity that also displayed pacemaker properties when glutamatergic, GABAergic, and glycinergic synaptic transmission were blocked with NBQX $(10 \mu \mathrm{M}),(\mathrm{PP}(10 \mu \mathrm{M})$, picrotoxin $(100 \mu \mathrm{M})$, and strychnine $(1 \mu \mathrm{M})$.

Glazkova, 2007). These injections produced small bursts of DIA $_{\mathrm{EMG}}$ activity during the IBI (Fig. $7 \mathrm{~A}$; burstlet normalized to burst amplitude: $0.16 \pm 0.05$ a.u., $n=4$ rats), similar to the burstlets recorded in vitro that leaked through to XII output in conditions of high excitability.

In addition to in vivo burstlets, we observed double-peaked bursts of DIA $\mathrm{EMG}_{\mathrm{EMG}}$ activity following bombesin and bicuculline injections that resembled doublets observed in vitro in XII motor output (Fig. 7A; Janczewski et al., 2012). These in vivo doublets were more reliably produced by blocking inhibition with local injection of bicuculline and the glycine receptor antagonist strychnine into the preBötC (Fig. 7B; Janczewski et al., 2012). This resulted in bursts with two peaks where the second peak was not significantly different from the first peak in $D_{\text {IIA }}$ (Fig. $7 B$; doublet second peak normalized to first peak: $0.74 \pm 0.09$ a.u.; paired $t$ test, $\left.t_{(3)}=2.6, p=0.08 ; n=4\right)$. The first peak of these doublets did not differ significantly from the amplitude of the single-peaked "eupneic" bursts (Fig. $7 B$; doublet first peak normalized to burst: $1.02 \pm 0.08$ a.u.; paired $t$ test, $t_{(3)}=0.23, p=$ $0.8 ; n=4)$. These in vivo burst shapes, with two equal peaks, seen

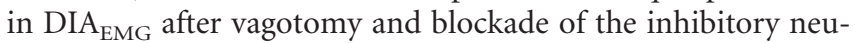
rotransmitters GABA and glycine (Fig. $7 B$ ), closely resembled XII doublet activity in vitro (Fig. 4A).

\section{Discussion}

In high excitability conditions, such as $9 \mathrm{~mm} \mathrm{~K}_{\text {ext }}^{+} / 1.5 \mathrm{~mm} \mathrm{Ca}_{\text {ext }}^{2+}$ in the slice preparation and presumably the resting state in vivo, the mammalian respiratory CPG generates a stable and robust motor output. In this mode in vitro, preBötC bursts are hypothesized to be rhythmogenic, i.e., necessary for determination of time to the next burst (Rekling et al., 1996; Feldman et al., 2013). We speculated that large safety factors and/or redundancies that may ensure robust inspiratory burst generation could make preBötC rhythmogenic mechanisms difficult to unravel from preBötC patterning mechanisms (Mellen, 2008, 2010; Marder, 2011). By lowering $\mathrm{K}_{\text {ext }}^{+}$from 9 to $6 \mathrm{mM}$, we increased variability in the XII burst rhythm. This resulted, not from a concurrent change in the timing of preBötC rhythmic activity, which did not differ across the two conditions, but from failures in preBötC burst generation and consequent XII burst generation, revealing distinct rhythm and pattern-generating mechanisms in vitro.

In slices bathed in $6 \mathrm{~mm} \mathrm{~K}_{\mathrm{ext}}^{+}$, XII activity displayed skipped bursts (Fig. 1), similar to "quantal slowing" seen in breathing rhythms following opiate administration in vitro and in vivo (Mellen et al., 2003; Janczewski and Feldman, 2006) and "deletions" in motor nerves during in vitro locomotion (Zhong et al., 2012). Such behavior is postulated to arise from the persistence of rhythmic activity in one (or more) rhythmogenic population(s) of neurons that drive nonrhythmogenic downstream premotoneuronal and motoneuronal pattern-generating networks (Mellen et al., 2003; Janczewski and Feldman, 2006; McCrea and Rybak, 2008). Here, rhythmic preBötC burstlet activity maintained timing information during skipped XII bursts (Fig. 2). Moreover, $\mathrm{T}_{\mathrm{XII}}$ increased monotonically with the number of burstlets and were distributed multimodally with longer $\mathrm{T}_{\mathrm{XII}}$ approximately equivalent to integer multiples of the interburstlet interval (Fig. 2D).

preBötC activity, however, was not just rhythmogenic. preBötC bursts, which were closely associated with XII bursts, occurred in phase with the burstlet rhythm yet were significantly larger than burstlets. The markedly lower amplitude of burstlets was not due to small, isolated clusters of active neurons. More than $93 \%$ of burstlets were bilaterally synchronous in population recordings and in simultaneous recordings of individual neurons and contralateral population activity (Figs. 2E, 5A). Burstlets observed unilaterally could result from failure to detect smaller amplitude burstlets due to noise. Burstlets, together with preBötC bursts, produced $\mathrm{T}_{\text {preBötC }}$ that were significantly more regular than $\mathrm{T}_{\mathrm{XII}}$. Burstlet $\rightarrow$ next event and burst $\rightarrow$ next event $\mathrm{T}_{\text {preBötC }}$ did not differ, suggesting that substantial additional activity during a preBötC I-burst did not significantly affect timing. With sufficient suprathreshold activity, the period defined by burstlet-mediated rhythmogenesis can be modified, as with the significantly longer doublet $\rightarrow$ next event $\mathrm{T}_{\text {preBötC }}$ (Fig. $4 B$ ) and the occasional longer burst $\rightarrow$ next event $\mathrm{T}_{\text {preBötC }}$ (Fig. $2 A, E)$. The preBötC, therefore, generates rhythmic activity and initiates its transformation into XII bursts to participate in pattern generation.

We suggest that preBötC bursts result from a low amplitude rhythmogenic preinspiratory component, resembling burstlets in rise time, duration, and peak amplitude, and a high amplitude pattern-generating I-burst. When preBötC bursts occurred, the rapidly rising preBötC I-burst obscured the falling phase of burstlets, so the burstlet appeared as preinspiratory activity. Conversely, the failure of rhythmogenic burstlets to produce XII output during skipped bursts resulted from the inability to engage preBötC I-burstgenerating, i.e., patterning, mechanisms.

This two-stage model of preBötC burst generation asserts that burstlet/preinspiratory activity is obligatory for I-bursts and, consequently, XII bursts. Indeed, triggering XII bursts with patterned photostimulation of small numbers (4-9) of 

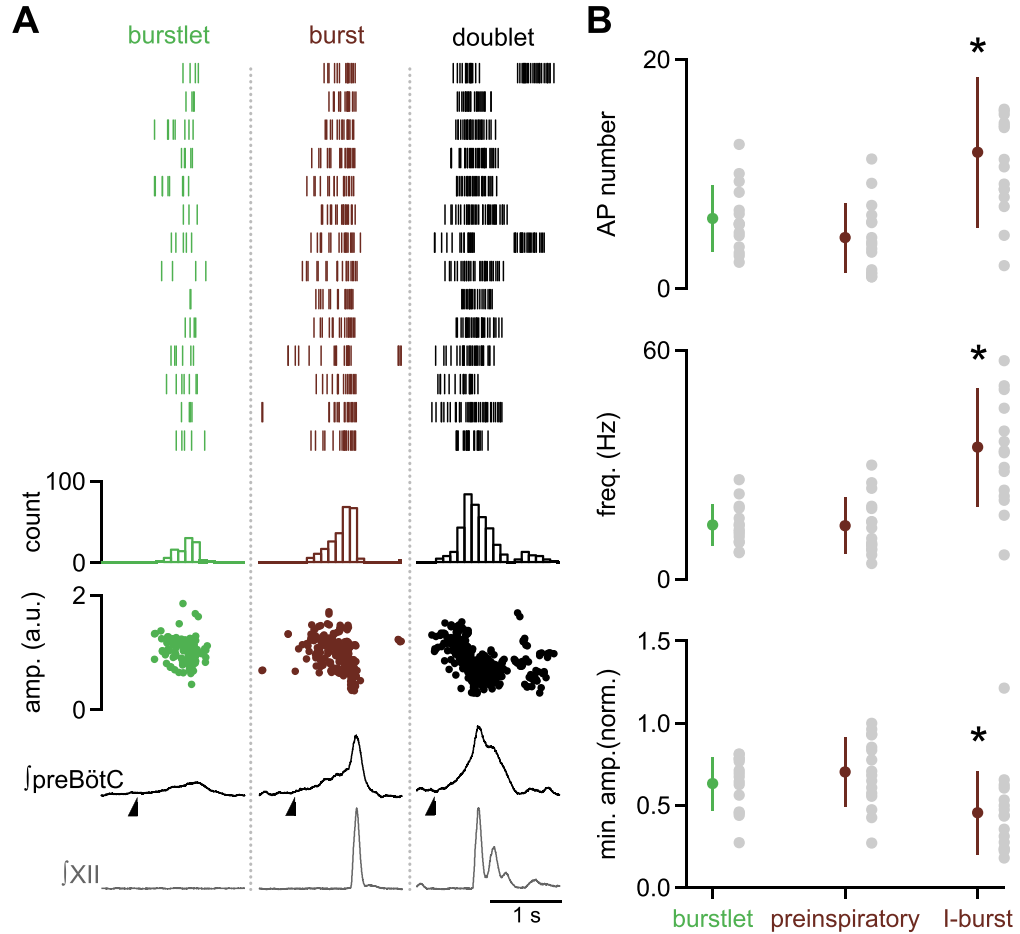

Figure 6. Characteristics of preBötC neuron firing patterns during burstlets and bursts. A, Top, APs during burstlets (left, green), bursts (middle, maroon), or doublets (right, black) of one preBötC inspiratory-modulated neuron. Each line of each column of the raster plot represents APs of recorded neuron during a single burstlet, burst, or doublet. $A$, Middle, Histograms of raster plots show the average AP frequency during burstlets, bursts, and doublets. A, Bottom, Amplitudes of APs over time during burstlets, bursts, and doublets are shown below the histograms. Time course of amplitude shows that AP waveform does not change significantly during burstlets, but decreases during bursts and doublets. After a pause in firing during some doublets, more APs are seen, corresponding to a second peak in the doublet. Raster plots, histograms, and amplitudes in a.u. are aligned to the average burstlet or XII burst/doublet waveform. Half-arrow indicates onset of preBötC burstlet, burst, and doublet. $B$, Comparison of firing pattern properties including AP number, frequency, and minimum amplitude normalized to the first AP in the event during burstlets (green), and the preinspiratory and I-burst components (maroon) of bursts in inspiratory-modulated neurons. Data are mean \pm SD $\left({ }^{*} p<0.05 ; n=16\right)$ with means for each slice as gray circles.

inspiratory-modulated preBötC neurons elicits ectopic XII bursts, resembling endogenous bursts, with an average latency of $255 \mathrm{~ms}$ (Kam et al., 2013), similar to the 100-400 ms duration of preinspiratory activity observed here and previously (Rekling et al., 1996). Increasing the number of targeted neurons decreases the latency between stimulation and XII burst initiation (Kam et al., 2013), just as increasing excitability decreased preinspiratory duration. We propose that onset of activity in a few preBötC neurons during the IBI is the key rhythmogenic event that seeds both burstlet and preinspiratory activity, which percolates through the network to trigger an I-burst by a distinct mechanism.

By recording extracellular activity from single preBötC neurons (Figs. 5, 6), we determined that low amplitude preBötC burstlet activity resulted from low-frequency firing in $\sim 90 \%$ of preBötC inspiratory-modulated neurons that also fired during bursts. No neurons fired only during burstlets. The increase in population activity during preBötC I-bursts resulted from significantly higher firing frequency in the same neurons generating burstlets plus a small $(\sim 10 \%)$ population of preBötC I-burst-only neurons. Whether preBötC I-burst-only neuron activity was necessary for generating preBötC I-bursts remains to be determined.

preBötC rhythm (burstlet) and pattern (I-burst) generation could be disassociated with $\mathrm{Cd}_{\text {ext }}^{2+} \cdot \mathrm{Cd}_{\text {ext }}^{2+}$ abolished preBötC bursts and all XII output, while leaving rhythmic burstlets intact (Fig. $3 E, F) . \mathrm{Cd}_{\text {ext }}^{2+}$, a broad spectrum blocker of voltage-gated $\mathrm{Ca}^{2+}$ channels that can reduce $\mathrm{Ca}^{2+}$-activated conductances, could affect preBötC I-burst generation by inhibiting activation of $\mathrm{I}_{\mathrm{CAN}}$ (ThobyBrisson and Ramirez, 2001) or reducing the efficacy of synaptic transmission between preBötC neurons (Reid et al., 1997).

Alternatively, several models of preBötC rhythmogenesis show rhythmic patterns similar to burstlet/burst rhythms, e.g., "irregular" or "intermittent" bursting in (Butera et al., 1999b; Rybak et al., 2004; Purvis et al., 2007), that suggest other mechanisms mediating preBötC I-burst generation. The regimes where "irregular" rhythms appear can be functions of the distribution of $\mathrm{I}_{\mathrm{NaP}}$ conductance (Rybak et al., 2004; Purvis et al., 2007) or of coupling strength (Butera et al., 1999b). Thus, lowering excitability may increase variability in $\mathrm{I}_{\mathrm{NaP}}$ across neurons or decrease coupling strength to produce a mixed burstlet/burst preBötC rhythm, suggesting that I-burst generation is sensitive to manipulations that decrease synchrony across the network.

preBötC bursts did not solely determine the pattern of XII activity. Increasing excitability could produce low levels of motor activity synchronous with burstlets, in vitro (XII; Fig. 4C) and in vivo (diaphragm; Fig. 7A), suggesting a tunable transmission threshold from preBötC to inspiratory motoneurons. This threshold is below peak preBötC burst activity as XII burst initiation occurred during the preBötC I-burst rise, and preBötC and XII bursts peaked almost concurrently. Additionally, some XII doublets exhibited a pause between peaks of activity that was not reflected in preBötC activity, which decreased, but did not reach baseline (Fig. 4A). This transmission threshold may involve preBötC I-burst-only neurons that, despite being a small minority, could function as an output or response-amplifying population, important in many networks (Feldt et al., 2011), or downstream premotoneurons or motoneurons, acting as high-pass amplitude filters that prevent lower levels of preBötC activity from reaching XII output (Chamberlin et al., 2007).

Measurement of motor output and, for in vitro preparations, acknowledgment of the absent role of sensory feedback in shaping motor pattern is critical for interpreting the role of interneuronal activity in the respiratory CPG. Solely recording preBötC population activity, small and large bursts observed in vitro were designated as "fictive" eupnea and sighs, respectively (Lieske et al., 2000; Ruangkittisakul et al., 2008). However, sighs, a doublepeaked inspiratory effort where a eupneic-like burst is followed closely by a larger second burst of activity (Cherniack et al., 1981; Orem and Trotter, 1993), were not observed in vivo in untreated vagotomized animals, even after increasing preBötC excitability (Fig. 7; Cherniack et al., 1981). Thus, sighs might not be expected to appear in medullary slices where sensory feedback is also absent. Additionally, "fictive" sighs (Lieske et al., 2000; Ruangkittisakul et al., 2008) are similar to preBötC bursts seen here, in both shape and sensitivity to $\mathrm{Cd}_{\mathrm{ext}}^{2+}$. How our burstlets and bursts map 
A

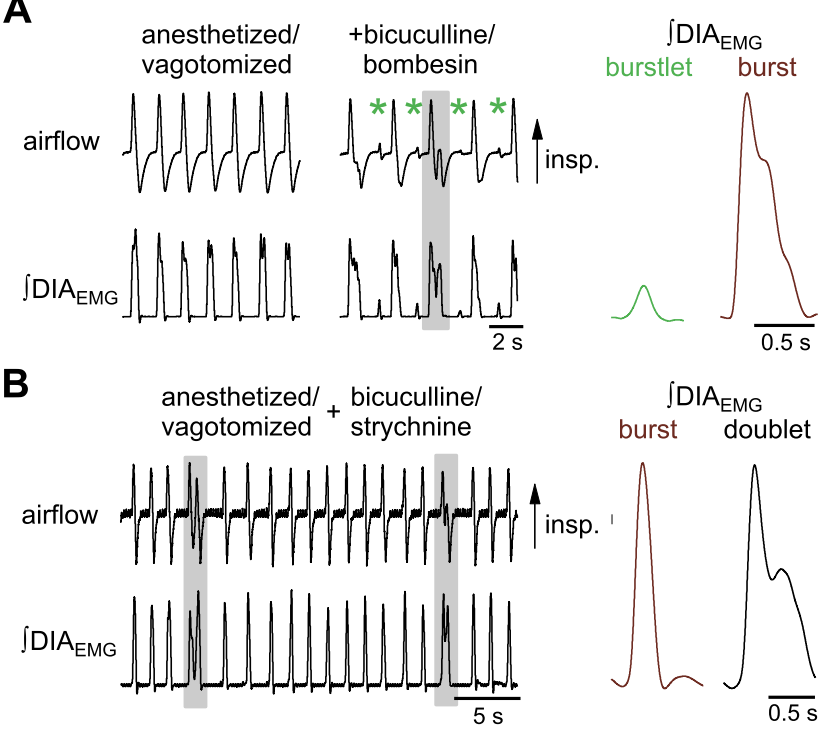

Figure 7. Burstlet/burst/doublet rhythms elicited in vivo. $A$, Respiratory pattern is shown in anesthetized and vagotomized adult rat before and after bicuculline injection into preBötC and BötC and bombesin injection into preBötC only. $A$, Left, Airflow and integrated DIA $A_{E M G}$ activity. After local injection of bicuculline and bombesin, small bursts (indicated by green asterisk) appeared between inspiratory bursts that resemble leak through of burstlets seen in vitro (Fig. $4 B)$. Doublets, e.g., gray box, were also observed. $A$, Right, Average integrated DIA ${ }_{E M G}$ during burstlets (green) and bursts (maroon). $\boldsymbol{B}$, Left, After vagotomy and injection of bicuculline and strychnine into preBötC, doublet bursts appeared. Gray boxes indicate doublets. $\boldsymbol{B}$, Right, Average integrated waveforms of DIA $\mathrm{EMG}_{\mathrm{EM}}$ from 20 bursts (maroon) and doublets (black) after vagotomy and injection of inhibitory antagonists. While doublet peaks were not significantly different, the second peak in the average doublet waveform appears smaller due to alignment of doublets by their onset and differences in the doublet intrapeak interval.

to "fictive" sighs and eupnea (Lieske et al., 2000; Ruangkittisakul et al., 2008) remains to be determined since recordings of simultaneous XII activity were absent in these prior studies, and experimental conditions differed.

Concurrent recordings of XII activity provided motor context to preBötC bursts and burstlets in vitro, but we also elicited burstlet-like motor activity in vivo by eliminating sensory feedback and increasing preBötC excitability, conditions intended to mimic those in the slice (Fig. 7). Our in vivo observations suggest that burstlets reflect processes also present in the adult intact rat, but not seen in motor output under normal conditions presumably due to a high safety factor for preBötC burstlets to ultimately trigger motor nerve bursts. Doublets were also observed in vivo. Although double breaths do not occur spontaneously in intact animals, we speculate that the two peaks of preBötC activity are transformed by sensory feedback and downstream patterngenerating networks to produce the classic augmented breath shape of sighs (Janczewski et al., 2012).

We conclude that distinct mechanisms within the preBötC underlie inspiratory rhythm and pattern generation. Separation of rhythm and pattern-generating mechanisms within the preBötC network provides a functional substrate for targeted modulation of either timing or pattern by other brain areas, e.g., raphé, retrotrapezoid nucleus, locus ceruleus, necessary to alter ventilation or affect breathing movements for various reflexive, emotive, and volitional respiratory-related behaviors. Because rhythmic bursting is a fundamental mode of nervous system activity, used in the encoding of multimodal sensory information and the execution of all forms of motor behavior, these mechanisms may be relevant for burst generation in many neural circuits.

\section{References}

Butera RJ Jr, Rinzel J, Smith JC (1999a) Models of respiratory rhythm generation in the pre-Bötzinger complex: I. Bursting pacemaker neurons. J Neurophysiol 82:382-397. Medline

Butera RJ Jr, Rinzel J, Smith JC (1999b) Models of respiratory rhythm generation in the pre-Bötzinger complex: II. Populations of coupled pacemaker neurons. J Neurophysiol 82:398-415. Medline

Chamberlin NL, Eikermann M, Fassbender P, White DP, Malhotra A (2007) Genioglossus premotoneurons and the negative pressure reflex in rats. J Physiol 579:515-526. CrossRef Medline

Cherniack NS, von Euler C, Glogowska M, Homma I (1981) Characteristics and rate of occurrence of spontaneous and provoked augmented breaths. Acta Physiol Scand 111:349-360. CrossRef Medline

Del Negro CA, Johnson SM, Butera RJ, Smith JC (2001) Models of respiratory rhythm generation in the pre-Bötzinger complex: III. Experimental tests of model predictions. J Neurophysiol 86:59-74. Medline

Del Negro CA, Morgado-Valle C, Hayes JA, Mackay DD, Pace RW, Crowder EA, Feldman JL (2005) Sodium and calcium current-mediated pacemaker neurons and respiratory rhythm generation. J Neurosci 25:446453. CrossRef Medline

Del Negro CA, Kam K, Hayes JA, Feldman JL (2009) Asymmetric control of inspiratory and expiratory phases by excitability in the respiratory network of neonatal mice in vitro. J Physiol 587:1217-1231. CrossRef Medline

Dobbins EG, Feldman JL (1994) Brainstem network controlling descending drive to phrenic motoneurons in rat. J Comp Neurol 347:64-86. CrossRef Medline

Dobbins EG, Feldman JL (1995) Differential innervation of protruder and retractor muscles of the tongue in rat. J Comp Neurol 357:376-394. CrossRef Medline

Feldman, JL (1986) Neurophysiology of breathing in mammals. In Handbook of physiology, Sec I, The nervous system, Vol IV, Intrinsic regulatory systems of the brain, (FE Bloom, ed), pp 463-524. Bethesda, MD: American Physiological Society.

Feldman JL, Del Negro CA, and Gray PA (2013) Understanding the rhythm of breathing: so near, yet so far. Annu Rev Physiol:75:423-452. CrossRef Medline

Feldt S, Bonifazi P, Cossart R (2011) Dissecting functional connectivity of neuronal microcircuits: experimental and theoretical insights. Trends Neurosci 34:225-236. CrossRef Medline

Gossard JP, Dubuc R, Kolta A (2010) The study of the neuronal networks: preface. Prog Brain Res 187:vii. CrossRef Medline

Gray PA, Rekling JC, Bocchiaro CM, Feldman JL (1999) Modulation of respiratory frequency by peptidergic input to rhythmogenic neurons in the preBötzinger complex. Science 286:1566-1568. CrossRef Medline

Gray PA, Janczewski WA, Mellen N, McCrimmon DR, Feldman JL (2001) Normal breathing requires preBötzinger complex neurokinin-1 receptorexpressing neurons. Nat Neurosci 4:927-930. CrossRef Medline

Hartigan JA, Hartigan PM (1985) The dip test of unimodality. Ann Stat 13:70-84. CrossRef

Iniushkin AN, Glazkova EN (2007) Respiratory effects of bombesin at the level of pre-Bötzinger complex in rats. Ross Fiziol Zh Im I M Sechenova 93:914-925. Medline

Janczewski WA, Feldman JL (2006) Distinct rhythm generators for inspiration and expiration in the juvenile rat. J Physiol 570:407-420. CrossRef Medline

Janczewski WA, Pagliardini S, Cui Y, and Feldman JL (2012) Sighing after vagotomy: program No. 796.05, 2012 neuroscience meeting planner. New Orleans: Society for Neuroscience.

Janczewski W, Tashima A, Hsu P, Cui Y, and Feldman JL (2013) Role of inhibition in respiratory pattern generation. J Neurosci 33:5454-5465. CrossRef Medline

Jarque CM, Bera AK (1987) A test for normality of observations and regression residuals. Int Stat Rev 55:163-172. CrossRef

Johnson SM, Koshiya N, Smith JC (2001) Isolation of the kernel for respiratory rhythm generation in a novel preparation: the pre-Bötzinger complex "island". J Neurophysiol 85:1772-1776. Medline

Kam K, Worrell JW, Ventalon C, Emiliani V, Feldman JL (2013) Emergence of population bursts from simultaneous activation of small subsets of preBötzinger complex inspiratory neurons. J Neurosci 33:3332-3338. CrossRef Medline

Koizumi H, Wilson CG, Wong S, Yamanishi T, Koshiya N, Smith JC (2008) 
Functional imaging, spatial reconstruction, and biophysical analysis of a respiratory motor circuit isolated in vitro. J Neurosci 28:2353-2365. CrossRef Medline

Lieske SP, Thoby-Brisson M, Telgkamp P, Ramirez JM (2000) Reconfiguration of the neural network controlling multiple breathing patterns: eupnea, sighs and gasps (see comment). Nat Neurosci 3:600-607. CrossRef Medline

Marder E (2011) Variability, compensation, and modulation in neurons and circuits. Proc Natl Acad Sci U S A 108:15542-15548. CrossRef Medline

McCrea DA, Rybak IA (2008) Organization of mammalian locomotor rhythm and pattern generation. Brain research reviews 57:134-146. CrossRef Medline

Mellen NM (2008) Belt-and-suspenders as a biological design principle. Adv Exp Med Biol 605:99-103. CrossRef Medline

Mellen NM (2010) Degeneracy as a substrate for respiratory regulation. Respir Physiol Neurobiol 172:1-7. CrossRef Medline

Mellen NM, Janczewski WA, Bocchiaro CM, Feldman JL (2003) Opioidinduced quantal slowing reveals dual networks for respiratory rhythm generation. Neuron 37:821-826. CrossRef Medline

Orem J, Trotter RH (1993) Medullary respiratory neuronal activity during augmented breaths in intact unanesthetized cats. J Appl Physiol 74:761769. Medline

Pace RW, Mackay DD, Feldman JL, Del Negro CA (2007) Role of persistent sodium current in mouse preBötzinger complex neurons and respiratory rhythm generation. J Physiol 580:485-496. CrossRef Medline

Peña F, Parkis MA, Tryba AK, Ramirez JM (2004) Differential contribution of pacemaker properties to the generation of respiratory rhythms during normoxia and hypoxia. Neuron 43:105-117. CrossRef Medline

Purvis LK, Smith JC, Koizumi H, Butera RJ (2007) Intrinsic bursters increase the robustness of rhythm generation in an excitatory network. J Neurophysiol 97:1515-1526. CrossRef Medline

Reid CA, Clements JD, Bekkers JM (1997) Nonuniform distribution of $\mathrm{Ca}^{2+}$ channel subtypes on presynaptic terminals of excitatory synapses in hippocampal cultures. J Neurosci 17:2738-2745. Medline

Rekling JC, Champagnat J, Denavit-Saubié M (1996) Electroresponsive properties and membrane potential trajectories of three types of inspiratory neurons in the newborn mouse brainstem in vitro. J Neurophysiol 75:795-810. Medline
Ruangkittisakul A, Secchia L, Bornes TD, Palathinkal DM, Ballanyi K (2007) Dependence on extracellular $\mathrm{Ca}^{2+} / \mathrm{K}+$ antagonism of inspiratory centre rhythms in slices and en bloc preparations of newborn rat brainstem. J Physiol 584:489-508. CrossRef Medline

Ruangkittisakul A, Schwarzacher SW, Secchia L, Ma Y, Bobocea N, Poon BY, Funk GD, Ballanyi K (2008) Generation of eupnea and sighs by a spatiochemically organized inspiratory network. J Neurosci 28:2447-2458. CrossRef Medline

Ruangkittisakul A, Panaitescu B, Ballanyi K (2011) K(+) and Ca(2) (+) dependence of inspiratory-related rhythm in novel "calibrated" mouse brainstem slices. Respir Physiol Neurobiol 175:37-48. CrossRef Medline

Rubin JE, Hayes JA, Mendenhall JL, Del Negro CA (2009) Calciumactivated nonspecific cation current and synaptic depression promote network-dependent burst oscillations. Proc Natl Acad Sci U S A 106: 2939-2944. CrossRef Medline

Rybak IA, Shevtsova NA, Ptak K, McCrimmon DR (2004) Intrinsic bursting activity in the pre-botzinger complex: role of persistent sodium and potassium currents. Biol Cybern 90:59-74. CrossRef Medline

Selverston AI (2010) Invertebrate central pattern generator circuits. Philos Trans R Soc Lond B Biol Sci 365:2329-2345. CrossRef Medline

Smith JC, Ellenberger HH, Ballanyi K, Richter DW, Feldman JL (1991) PreBötzinger complex: a brainstem region that may generate respiratory rhythm in mammals. Science 254:726-729. CrossRef Medline

Tan W, Janczewski WA, Yang P, Shao XM, Callaway EM, Feldman JL (2008) Silencing preBötzinger complex somatostatin-expressing neurons induces persistent apnea in awake rat. Nat Neurosci 11:538-540. CrossRef Medline

Tan W, Sherman D, Turesson J, Shao XM, Janczewski WA, Feldman JL (2012) Reelin demarcates a subset of pre-Bötzinger complex neurons in adult rat. J Comp Neurol 520:606-619. CrossRef Medline

Thoby-Brisson M, Ramirez JM (2001) Identification of two types of inspiratory pacemaker neurons in the isolated respiratory neural network of mice. J Neurophysiol 86:104-112. Medline

Zhong G, Shevtsova NA, Rybak IA, Harris-Warrick RM (2012) Neuronal activity in the isolated mouse spinal cord during spontaneous deletions in fictive locomotion: insights into locomotor central pattern generator organization. J Physiol 590:4735-4759. CrossRef Medline 